Louisiana State University

LSU Digital Commons

$11-1-2015$

\title{
The ages of a-stars. I. Interferometric observations and age estimates for stars in the ursa major moving group
}

\author{
Jeremy Jones \\ Georgia State University \\ R. J. White \\ Georgia State University \\ T. Boyajian \\ Yale University \\ G. Schaefer \\ Georgia State University \\ E. Baines \\ Naval Research Laboratory
}

See next page for additional authors

Follow this and additional works at: https://digitalcommons.Isu.edu/physics_astronomy_pubs

\section{Recommended Citation}

Jones, J., White, R., Boyajian, T., Schaefer, G., Baines, E., Ireland, M., Patience, J., Brummelaar, T., McAlister, H., Ridgway, S., Sturmann, J., Sturmann, L., Turner, N., Farrington, C., \& Goldfinger, P. (2015). The ages of astars. I. Interferometric observations and age estimates for stars in the ursa major moving group. Astrophysical Journal, 813 (1) https://doi.org/10.1088/0004-637X/813/1/58 


\section{Authors}

Jeremy Jones, R. J. White, T. Boyajian, G. Schaefer, E. Baines, M. Ireland, J. Patience, T. Ten Brummelaar, H. McAlister, S. T. Ridgway, J. Sturmann, L. Sturmann, N. Turner, C. Farrington, and P. J. Goldfinger 


\title{
THE AGES OF A-STARS. I. INTERFEROMETRIC OBSERVATIONS AND AGE ESTIMATES FOR STARS IN THE URSA MAJOR MOVING GROUP
}

\author{
Jeremy Jones $^{1}$, R. J. White ${ }^{1}$, T. Boyajian $^{2}$, G. Schaefer ${ }^{1}$, E. Baines ${ }^{3}$, M. Ireland ${ }^{4}$, J. Patience ${ }^{5}$, T. Ten BrummelaAR ${ }^{1}$, \\ H. McAlister ${ }^{1}$, S. T. Ridgway ${ }^{6}$, J. Sturmann ${ }^{1}$, L. Sturmann ${ }^{1}$, N. Turner ${ }^{1}$, C. Farrington ${ }^{1}$, And P. J. Goldfinger ${ }^{1}$ \\ ${ }^{1}$ Center for High Angular Resolution Astronomy and Department of Physics and Astronomy, Georgia State University, \\ 25 Park Place, Suite 605, Atlanta, GA 30303, USA; jones@ astro.gsu.edu \\ ${ }^{2}$ Department of Astronomy, Yale University, New Haven, CT 06511, USA \\ ${ }^{3}$ Remote Sensing Division, Naval Research Laboratory, 4555 Overlook Avenue SW, Washington, DC 20375, USA \\ ${ }^{4}$ Research School of Astronomy \& Astrophysics, Australian National University, Canberra ACT 2611, Australia \\ ${ }^{5}$ School of Earth and Space Exploration, Arizona State University, P.O. Box 871404, Tempe, AZ 85287, USA \\ ${ }^{6}$ National Optical Astronomy Observatory, 950 North Cherry Avenue, Tucson, AZ 85719, USA \\ Received 2015 April 29; accepted 2015 August 21; published 2015 October 28
}

\begin{abstract}
We have observed and spatially resolved a set of seven A-type stars in the nearby Ursa Major moving group with the Classic, CLIMB, and PAVO beam combiners on the Center for High Angular Resolution Astronomy Array. At least four of these stars have large rotational velocities $\left(v \sin i \gtrsim 170 \mathrm{~km} \mathrm{~s}^{-1}\right)$ and are expected to be oblate. These interferometric measurements, the stars' observed photometric energy distributions, and $v \sin i$ values are used to computationally construct model oblate stars from which stellar properties (inclination, rotational velocity, and the radius and effective temperature as a function of latitude, etc.) are determined. The results are compared with MESA stellar evolution models to determine masses and ages. The value of this new technique is that it enables the estimation of the fundamental properties of rapidly rotating stars without the need to fully image the star. It can thus be applied to stars with sizes comparable to the interferometric resolution limit as opposed to those that are several times larger than the limit. Under the assumption of coevality, the spread in ages can be used as a test of both the prescription presented here and the MESA evolutionary code for rapidly rotating stars. With our validated technique, we combine these age estimates and determine the age of the moving group to be $414 \pm 23 \mathrm{Myr}$, which is consistent with, but much more precise than previous estimates.
\end{abstract}

Key words: stars: early-type - stars: evolution - stars: rotation - techniques: interferometric

Supporting material: tar.gz file

\section{INTRODUCTION AND MOTIVATION}

Population I stars with spectral type A have masses that range from 1.5 to $2.5 M_{\odot}$, based on dynamical measurements of spectroscopic binaries (e.g., Torres et al. 2010), and corresponding main sequence lifetimes of 3.6-1.0 Gyr (assuming $\tau_{\text {MS }} \propto M^{-2.5}$, Kippenhahn et al. 2012). However, measuring the age and mass of any single A-type star is often severely complicated by their peculiar characteristics, including chemically anomalous photospheres (e.g., Am stars, Ap stars, $\lambda$ Boo stars), radial and non-radial pulsations (e.g., $\gamma$ Doradus-type, $\delta$ Scuti-type), and severely distorted shapes from rapid rotation (e.g., Regulus-McAlister et al. 2005, Altair-Monnier et al. 2007, Vega-Aufdenberg et al. 2006; Monnier et al. 2012). Despite these challenges, interest in determining precise ages and masses for A-type stars has been heightened with the discoveries of directly imaged candidate planets orbiting several of them, including HR8799 (Marois et al. 2008); Fomalhaut (Kalas et al. 2008); etc. In all cases the estimated masses of the planetary companions depend critically on the ages assigned to the host stars (e.g., Moya et al. 2010 versus Marois et al. 2010), which in most cases are poorly determined.

Although a variety of independent techniques exist for estimating the ages of Sun-like stars, such as photospheric lithium depletion or chromospheric activity (e.g., Mamajek \& Hillenbrand 2008), these are ineffective for A-type stars with predominantly radiative atmospheres. For these stars, a more effective method is to compare observable stellar properties (e.g., radius, luminosity, and temperature) to the predictions of stellar evolutionary models (e.g., Brandt \& Huang 2015; David \& Hillenbrand 2015). This "isochronal fitting" technique has the potential to work well for A-type stars since their radii, temperatures, and luminosities evolve much more substantially than Sun-like stars do during the first $\sim \mathrm{Gyr}$ of their main sequence lifetime. For example, the MESA evolutionary models (Paxton et al. 2011, 2013) predict that the radius, luminosity, and temperature of a $2 M_{\odot}$ star change by $+32 \%$, $+20 \%$, and $-10 \%$, respectively, in just $500 \mathrm{Myr}$ after the zeroage main sequence. ${ }^{7}$ This can be compared to a $1 M_{\odot}$ star that, in the same time frame sees its radius, luminosity, and temperature change by only $+3.0 \%,+8.8 \%$, and $+0.6 \%$, respectively. Figure 1 illustrates these differences in evolutionary rates with four stars with masses between 1 and $2.5 M_{\odot}$.

Unfortunately, the peculiar properties of A-stars make these relatively straight-forward comparisons with models difficult in practice. Pulsation and rapid rotation result in observable stellar properties that are both time and orientation dependent. While the photometric variations due to pulsations are typically less than a few percent (Henry et al. 2007), rapid rotation distends the star so that its size is no longer defined by a single radius. The resulting gravity darkening that occurs creates a temperature gradient on the star's surface (von Zeipel 1924a, 1924b), causing the star to no longer be defined by a single temperature.

\footnotetext{
The zero-age main sequence is defined for each star to be the point at which the contribution to the luminosity of the star due to gravitational contraction is $\sim 1 \%$ that ofcore fusion as predicted by the MESA evolution code.
} 

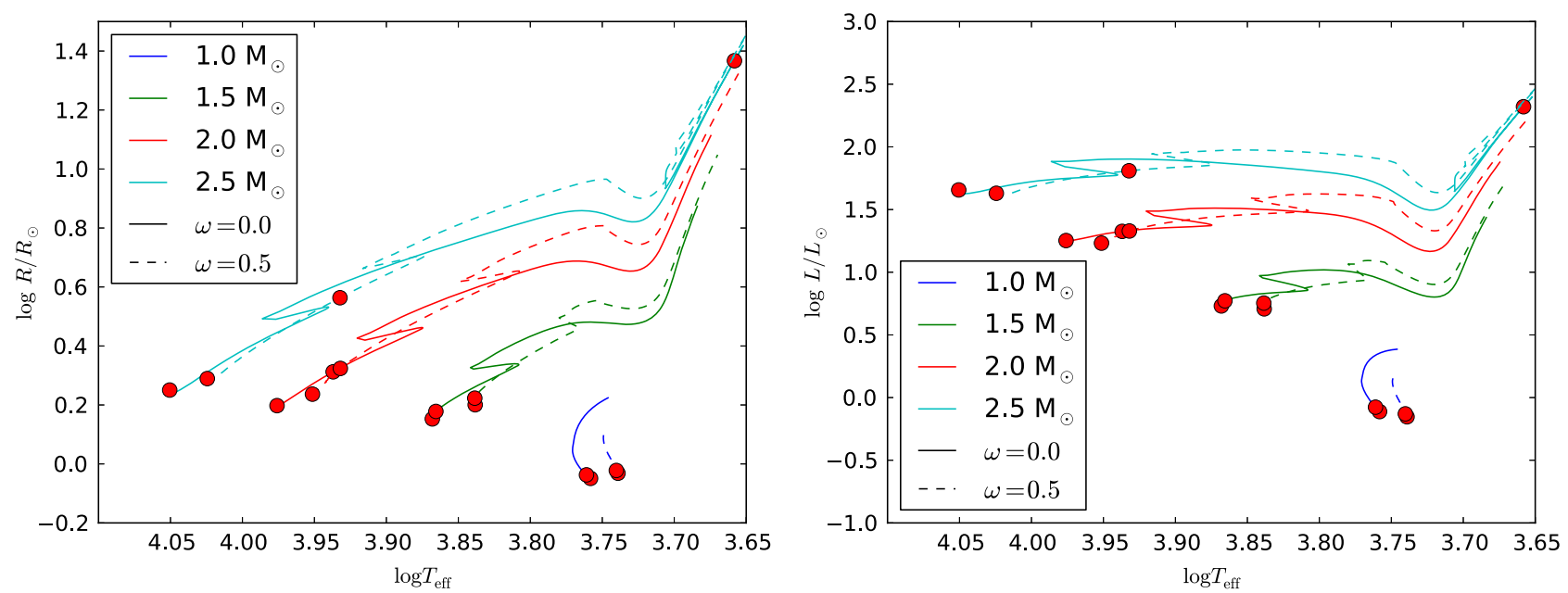

Figure 1. Plot of temperature vs. radius (left) and temperature vs. luminosity (right) of the evolution tracks of eight stars with masses ranging from 1.0 to $2.5 M_{\odot}$ and an angular rotation rate of either $0 \%$ or $50 \%$ that of the break-up velocity. The red circles represent the properties of each star while on the zero age main sequence (at 41, 22, 9.5, and 5.7 Myr for the 1.0, 1.5, 2.0 and 2.5 $M_{\odot}$ stars, respectively, for $\omega=0.0$ and 49, 26, 11, and 5.9 Myr for $\left.\omega=0.5\right)$ and 500 Myr after that point.

The net effect is that the observed flux depends on the star's inclination, making the total luminosity challenging to determine since inclination is unknown for most stars. In addition to the challenges in observationally determining an A-star's stellar properties, their peculiar characteristics must be accounted for in the adopted stellar evolution models. Rapid rotation has been shown to dramatically affect the way the star evolves (Meynet \& Maeder 2000; Maeder \& Meynet 2010). For example, the MESA evolutionary code predicts that solarto intermediate-mass stars rotating at $50 \%$ of break-up velocity have average surface temperatures that are significantly cooler relative to non-rotating stars, and evolve more slowly off the main sequence (Figure 1). Finally, the anomalous surface abundances of many A-stars can complicate the choice of evolutionary model metallicity, which is usually scaled relative to solar.

Fortunately, with the high angular resolution that optical/ infrared interferometers provide, it is now possible to use interferometric imaging, often referred to as aperture synthesis (Baron et al. 2010), to directly determine fundamental properties of rapidly rotating early-type stars (Che et al. 2011; Monnier et al. 2012; van Belle 2012). In these cases, the oblateness and gravity darkening can be observed directly, which enables more accurate determination of the star's luminosity and comparisons with evolutionary models. However, there are only a handful of stars that are large enough and bright enough for this technique to work effectively with current facilities.

We present a technique that allows for the correction of the effects of rotational distortion without having to fully image the star. Fundamental parameters are determined by tuning a model of an observed rapidly rotating star such that the modelcalculated interferometric visibilities match the observed visibilities obtained at multiple baseline orientations; the model is further constrained by the star's photometric energy distribution (PED) and projected rotational velocity $(v \sin i)$. The advantage of this technique is that it enables the determination of fundamental properties of rapidly rotating stars that are too small and/or too faint to be observed with imaging interferometric beam combiners. Of the 112 A-type stars within 50 parsecs that are observable with the Center for High Angular Resolution Astronomy (CHARA) Array (i.e.,
Table 1

Age Estimates for the Ursa Major Moving Group

\begin{tabular}{lc}
\hline \hline $\begin{array}{l}\text { Age } \\
(\mathrm{Myr})\end{array}$ & Reference \\
\hline$\sim 300$ & von Hoerner (1957) \\
$300 \pm 100$ & Giannuzzi (1979) \\
$630-1000$ & Eggen (1992) \\
$300-400$ & Soderblom et al. (1993) \\
$\sim 500$ & Asiain et al. (1999) \\
$\sim 200$ & König et al. (2002) \\
$500 \pm 100$ & King et al. (2003) \\
$\sim 600$ & King \& Schuler (2005) \\
$393^{\mathrm{a}}$ & David \& Hillenbrand (2015) \\
$530 \pm 40$ & Brandt \& Huang (2015) \\
$414 \pm 23$ & This work \\
\hline
\end{tabular}

Note.

${ }^{a}$ David \& Hillenbrand (2015) do not report an age for the UMa moving group. The value listed here corresponds to the median of the ages they report for the seven Ursa Majoris stars studied here (Table 2).

with $\delta>-10^{\circ}$ and with no known companions within $2^{\prime \prime}$ and with $\Delta M_{V}<5 \mathrm{mag}$ ), only 13 have estimated angular diameters large enough $(\theta \gtrsim 1$ mas) to fully benefit from imaging. Another advantage of this technique is that it does not require the measurement of closure phases, so it is not necessary to use the many simultaneous baselines that are necessary for the aperture synthesis imaging technique. In this paper, we demonstrate the success of this technique by comparing the relative ages of rapidly and non-rapidly rotating stars in the Ursa Major moving group. These ages are determined by comparing modeled luminosities and radii with the predictions of the MESA evolution model. Indirectly, the results thus also provide a new age estimate for this moving group and tests of gravity darkening laws and stellar evolutionary models that include rotation.

\section{THE SAMPLE AND CHARA OBSERVATIONS}

With a nucleus distance of $25 \mathrm{pc}$, the Ursa Major moving group is one of the closest and best-studied moving groups. It consists of 15 nucleus stars and 47 likely stream members with 
Table 2

Presented Sample

\begin{tabular}{|c|c|c|c|c|c|c|c|c|c|}
\hline $\begin{array}{l}\text { Common } \\
\text { Name }\end{array}$ & $\begin{array}{c}\text { HD } \\
\text { Number }\end{array}$ & $\begin{array}{c}\text { HIP } \\
\text { Number }\end{array}$ & $\begin{array}{l}\text { Spectral } \\
\text { Type }^{\mathrm{a}}\end{array}$ & $\begin{array}{c}v \sin i^{\mathrm{b}} \\
\left(\mathrm{km} \mathrm{s}^{-1}\right)\end{array}$ & $\begin{array}{l}D^{\mathrm{c}} \\
(\mathrm{pc})\end{array}$ & $\begin{array}{c}V_{\mathrm{T}}^{\mathrm{d}} \\
(\mathrm{mag})\end{array}$ & $\begin{array}{c}B-V^{\mathrm{d}} \\
(\mathrm{mag})\end{array}$ & $\begin{array}{c}K_{S}^{\mathrm{e}} \\
(\mathrm{mag})\end{array}$ & $\begin{array}{c}\text { UMa } \\
\text { Membership }^{\mathrm{f}}\end{array}$ \\
\hline Merak & 95418 & 53910 & A1 IVps (SrII) & $46 \pm 2.3$ & $24.4 \pm 0.1$ & 2.35 & 0.033 & 2.285 & Nuclear \\
\hline Phecda & 103287 & 58001 & A1 IV(n) & $178 \pm 8.9$ & $25.5 \pm 0.3$ & 2.43 & 0.044 & 2.429 & Nuclear \\
\hline Megrez & 106591 & 59774 & $\mathrm{~A} 2 \mathrm{Vn}$ & $233 \pm 11.7$ & $24.7 \pm 0.1$ & 3.34 & 0.077 & 3.104 & Nuclear \\
\hline Alcor & 116842 & 65477 & A6 Vnn & $228 \pm 11.4$ & $25.1 \pm 0.1$ & 4.05 & 0.169 & 3.145 & Nuclear \\
\hline Chow & 141003 & 77233 & $\mathrm{~A} 2 \mathrm{~V}$ & $207 \pm 10.4$ & $47.6 \pm 0.6$ & 3.68 & 0.073 & 3.546 & Stream \\
\hline $16 \mathrm{Lyr}$ & 177196 & 93408 & A7: V & $124 \pm 6.2$ & $37.4 \pm 0.2$ & 5.07 & 0.186 & 4.505 & Stream \\
\hline 59 Dra & 180777 & 94083 & F0 Vs & $70 \pm 3.5^{\mathrm{g}}$ & $27.3 \pm 0.1$ & 5.19 & 0.308 & 4.313 & Stream \\
\hline
\end{tabular}

Notes.

a Nucleus Stars-Gray et al. (2003), Stream Stars-Levato \& Abt (1978).

${ }^{b}$ Royer et al. (2007).

c van Leeuwen (2007).

d Perryman et al. (1997).

e Cutri et al. (2003).

${ }^{\mathrm{f}}$ King et al. (2003).

g Glebocki \& Gnacinski (2005).
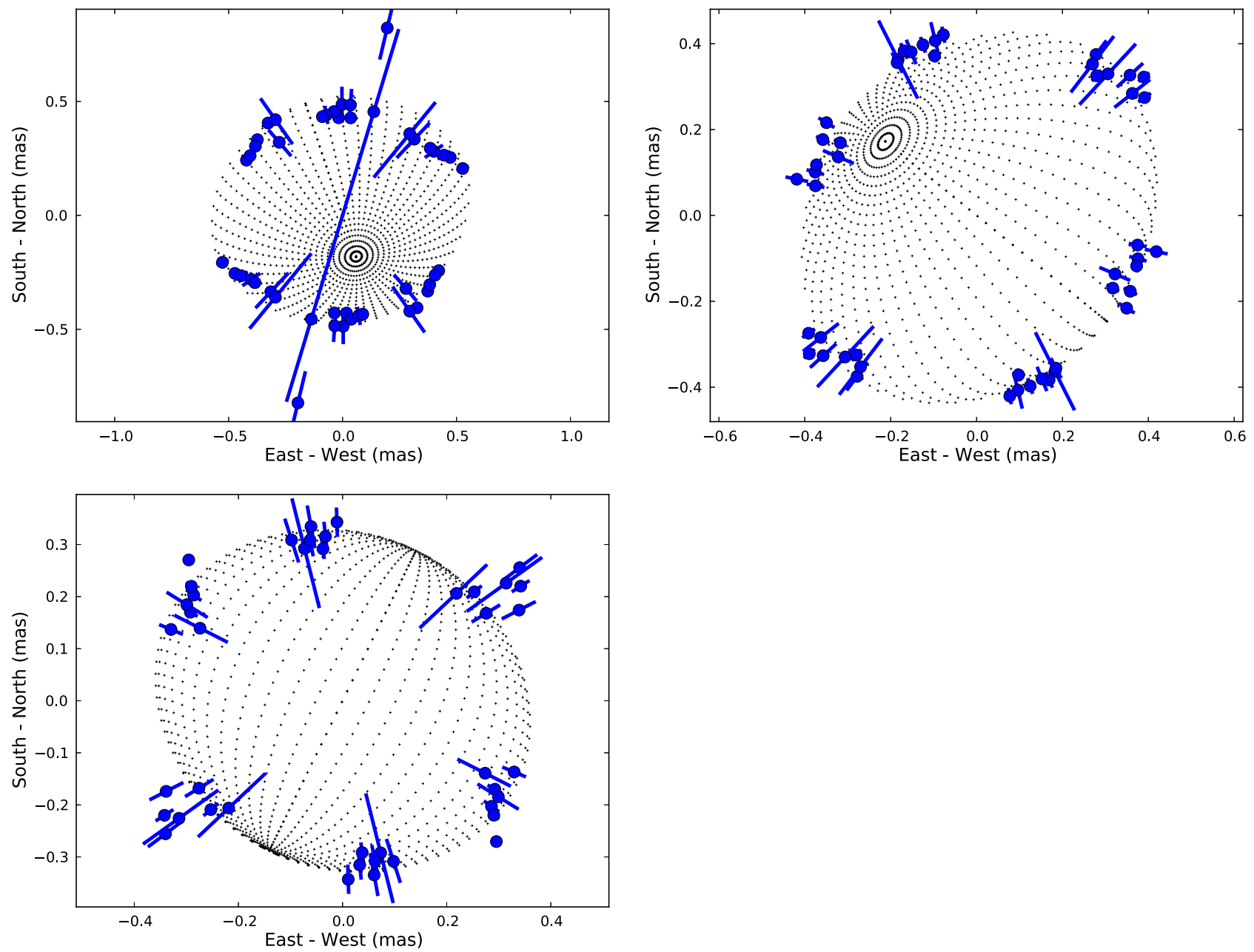

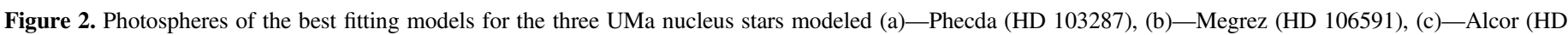

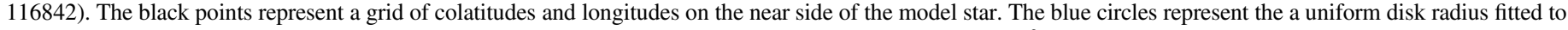
each individual visibility at the appropriate baseline orientation observed. The data are duplicated at a $180^{\circ}$ orientation.

an estimated age of $500 \pm 100 \mathrm{Myr}$ and a metallicity of $Z=0.016$ (King et al. 2003). As summarized in Table 1, previous studies have found an age for the moving group ranging from 200 to 1000 Myr. The introduction of Ammler- von Eiff \& Guenther (2009) provides an excellent history of the study of the UMa moving group.

We define a sample of A-stars in the Ursa Major moving group for interferometric observations by selecting all stars 

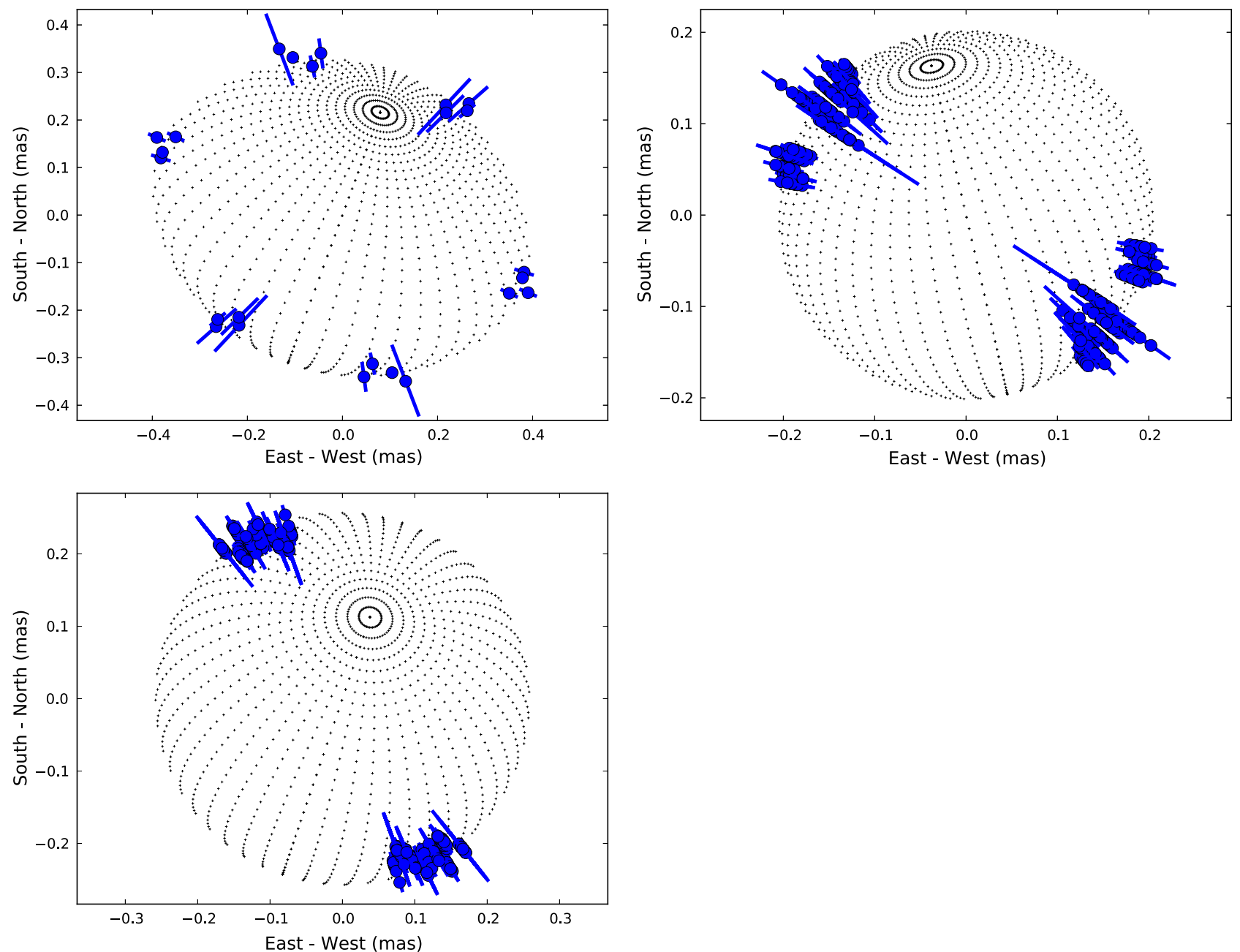

Figure 3. Same as Figure 2, but for the three UMa stream stars modeled: (a)—Chow (HD 141003), (b)—16 Lyr (HD 177196), (c) — 59 Dra (HD 180777). The baseline orientations of $16 \mathrm{Lyr}$ and 59 Dra are undersampled, making it difficult to measure their oblateness directly.

with $B-V$ colors less than 0.31 from the "UMa nucleus stars" list in King et al. (2003). The hottest of these stars, has a $B-V$ color of -0.022 (van Leeuwen 2007) and an assigned spectral type of A1 (Gray et al. 2003). The resulting list consists of seven stars of which two stars (Mizar A = HD 116656 and Mizar B $=$ HD 116657) form a spectroscopic binary pair of comparable brightness $\left(\Delta M_{V}=1.68 \mathrm{mag}\right)$. Mizar A and B are consequently excluded from this sample because the close proximity ( $\sim 4$ mas) and small $\Delta M_{V}$ of this pair would bias interferometric observations, making it difficult to distinguish the physical properties of each star individually. Another of these seven nucleus stars (Alioth $=$ HD 112185) has a possible companion star. Roberts (2011) identify a companion to Alioth with a projected separation of 0 !" 11 and a $\Delta M_{I}$ of 2.31 mag. A fourth of these seven stars (Alcor $=$ HD 116842) has an observed stellar companion of spectral type M3-M4 and with a projected separation of 1"'11 (Mamajek et al. 2010; Zimmerman et al. 2010). However, with a $\Delta M_{H}$ of $\sim 6$, the companion is too faint to contaminate the interferometric observations, so it is not excluded from the sample. None of the other nucleus stars have known companions (De Rosa et al. 2014). The four nucleus member stars that are included in this sample are Merak $=$ HD 95418, Phecda $=$ HD 103287, Megrez $=$ HD 106591, and Alcor $=$ HD 116842.
There are six additional A-stars that are likely stream members of the moving group (listed as "Y" or "Y?" in King et al. 2003). Two of these six (Menkalinan = HD 40183 and Alphecca $=$ HD 139006) are spectroscopic binaries with $\Delta M_{V}$ values of $\sim 1$ and $\sim 4$, respectively (Tomkin \& Popper 1986; Pourbaix 2000) and so are not observed. Of the remaining four, one star (21 LMi = HD 87696) was not observed due to limited telescope time. The remaining three (Chow $=$ HD 141003, 16 $\mathrm{Lyr}=\mathrm{HD} 177196$, and $59 \mathrm{Dra}=\mathrm{HD}$ 180777) are included in the sample. One of these stream stars (59 Dra) has a candidate brown dwarf companion (Galland et al. 2006), but this is too faint to contaminate the interferometric observations.

In total, we obtained new interferometric observations for six Ursa Major A-type stars (three nuclear members and three stream members). One additional star, Merak, was observed interferometrically by a previous study (Boyajian et al. 2012). These seven stars have spectral types ranging from A0 to A7. Merak also has a peculiar metallicity (Royer et al. 2014) and is an apparent slow rotator with a $v \sin i$ of $46 \pm 2.3 \mathrm{~km} \mathrm{~s}^{-1}$. While it is possible that Merak is a rapidly rotating star oriented pole-on, there is some suggestion that the peculiar metallicity of Ap stars is due in part to their slow rotation (Abt 2009, and references therein). Another apparent slow rotator in the observed sample is 59 Dra with a $v \sin i$ of $70 \pm 3.5 \mathrm{~km} \mathrm{~s}^{-1}$. 59 Dra shows a normal A-star metallicity suggesting that it may 
Table 3

Observing Log

\begin{tabular}{|c|c|c|c|c|c|c|c|c|}
\hline Target Name/HD & Cal HD & Cal Diameter (mas) & Combiner & Baseline & Bandpass & \# Brackets & \# Visibilities & Date \\
\hline \multirow{2}{*}{103287} & 99913 & $0.582 \pm 0.058$ & CLIMB & S2-E2-W2 & $K$ & 2 & 6 & 2012 Jun 2 \\
\hline & 105525 & $0.392 \pm 0.039$ & CLIMB & S1-E1-W1 & $K$ & 2 & 6 & 2013 May 11 \\
\hline Megrez & 108954 & $0.451 \pm 0.045$ & CLIMB & S1-E1-W1 & $H$ & 4 & 12 & 2012 Apr 20 \\
\hline 106591 & 108845 & $0.481 \pm 0.048$ & CLIMB & S1-E1-W1 & $H$ & 2 & 6 & 2012 Apr 21 \\
\hline$\overline{\text { Alcor }}$ & 119024 & $0.306 \pm 0.031$ & CLIMB & S1-E1-W1 & $H$ & 4 & 12 & 2012 Apr 20 \\
\hline \multirow[t]{2}{*}{116842} & 108954 & $0.451 \pm 0.045$ & CLIMB & S1-E1-W1 & $H$ & 1 & 3 & 2012 Apr 21 \\
\hline & 118232 & $0.465 \pm 0.047$ & CLIMB & S1-E1-W1 & $H$ & 2 & 6 & 2012 Apr 21 \\
\hline Chow & 140160 & $0.293 \pm 0.029$ & CLIMB & S1-E1-W1 & $H$ & 2 & 6 & 2012 Apr 21 \\
\hline 141003 & 137510 & $0.525 \pm 0.053$ & CLIMB & S1-E1-W1 & $H$ & 2 & 6 & 2012 Apr 21 \\
\hline & 177003 & $0.156 \pm 0.016$ & PAVO & E1-W2 & $R$ & 3 & 69 & 2013 Aug 5 \\
\hline & 185872 & $0.256 \pm 0.026$ & PAVO & E1-W1 & $R$ & 2 & 46 & 2013 Aug 5 \\
\hline 59 Dra & 184102 & $0.263 \pm 0.026$ & PAVO & S2-E2 & $R$ & 3 & 69 & $2012 \mathrm{Jul} 10$ \\
\hline \multirow[t]{3}{*}{180777} & 201908 & $0.187 \pm 0.019$ & PAVO & $\mathrm{S} 2-\mathrm{E} 2$ & $R$ & 3 & 69 & $2012 \mathrm{Jul} 10$ \\
\hline & 184102 & $0.263 \pm 0.026$ & PAVO & E2-W2 & $R$ & 3 & 69 & 2013 Aug 4 \\
\hline & 201908 & $0.187 \pm 0.019$ & PAVO & E2-W2 & $R$ & 3 & 69 & 2013 Aug 4 \\
\hline
\end{tabular}

be a rapidly rotating star oriented pole-on. The four stars in this set that are nuclear members have distances within the very narrow range of $24.4-25.5 \mathrm{pc}$, while the three stream members are more spread out, having distances of 27.3, 37.4, and $47.6 \mathrm{pc}$. The properties of all seven stars in the set are summarized in Table 2, which includes spectral type, projected rotational velocity, Hipparcos distance, photometry, and UMa membership as determined by King et al. (2003).

All observations were obtained using Georgia State University's CHARA Array. The CHARA Array is a six telescope interferometer which operates at optical and nearinfrared wavelengths (ten Brummelaar et al. 2005). The CHARA Array's six telescopes are arranged in a Y-shaped configuration with baselines ranging from 34 to $331 \mathrm{~m}$. The naming convention for these six telescopes consists of a letter representing one of three arms of the "Y" ("S" for south, "E" for east, and " $\mathrm{W}$ " for west), and a number indicating the outer telescope (1) or the inner telescope (2) of each arm. Data were obtained using three beam combiners: Classic, CLIMB, and PAVO. All three beam combiners measure the contrast of the interference pattern produced by the light from each of the telescopes used. This contrast is known as a visibility. The twotelescope Classic beam combiner takes a single visibility measurement per observation in a broadband near-infrared filter ( $K$-band for this work). The three-telescope CLIMB beam combiner, which also operates in the near-infrared (in either the $H$ - or $K$-band), takes three simultaneous visibility measurements for each broadband observation (one for each combination of two telescopes). The PAVO beam combiner was used in its two-telescope mode and each observation yields 23 visibilities spectrally dispersed across a wavelengths ranging from 0.65 to $0.79 \mu \mathrm{m}$. Because PAVO and Classic observations were taken using two telescopes at a time, only a narrow range of baseline orientations was used. This is illustrated in Figures 2-3. We note that for two stars (16 Lyr and 59 Dra), we do not have sufficient baseline orientations to measure oblateness. A general observing strategy was adopted whereby calibrator stars (described in Section 3) were observed both before and after each target star. This set of observations is referred to as a visibility bracket. Over eight nights of observing, a total of 56 visibility brackets yielding 724 individual visibility measurements were obtained on six stars. Boyajian et al. (2012) obtained 25 brackets on Merak with the two-telescope Classic beam combiner. Table 3 lists the calibrators, beam combiners, baselines, and wavelengths used during each observation as well as how many brackets were obtained for each star.

\section{DATA REDUCTION AND CALIBRATED VISIBILITIES}

Interferometric data from the Classic and CLIMB beam combiners were reduced using the redclassic and redclimb pipelines, respectively (ten Brummelaar et al. 2013), yielding reduced visibilities for each observation made. The pipeline used to reduce the observations made with the PAVO beam combiner is described by Ireland et al. (2008). Many factors, both atmospheric and instrumental, serve to decrease the visibility measured by an interferometer. This decrease depends in part on atmospheric turbulence at the time of observation and the airmass at which the star is observed (e.g., Roddier 1981, p. 281; Boden 2007). Correcting for these temporal effects on the visibility requires frequent observation of a star with a known angular diameter that is ideally smaller than the interferometric resolution $(\lambda / 2 B)$. Such a star is called a calibrator star. When observed near the target star both in time $(\lesssim 30$ minutes $)$ and on the sky $\left(\lesssim 10^{\circ}\right)$, the target star's intrinsic visibility $\left(V_{\mathrm{i}}^{*}\right)$ should be observed $\left(V_{\mathrm{m}}^{*}\right)$ to be reduced by the same amount as the 
Table 4

Model Results Using the vZ Gravity Darkening Law

\begin{tabular}{|c|c|c|c|c|c|c|}
\hline & Phecda & Megrez & Alcor & Chow & $16 \mathrm{Lyr}$ & 59 Dra \\
\hline HD Number & 103287 & 106591 & 116842 & 141003 & 177196 & 180777 \\
\hline Equatorial radius, $R_{\mathrm{e}}\left(R_{\odot}\right)$ & $3.435_{-0.148}^{+0.154}$ & $2.512_{-0.076}^{+0.075}$ & $2.002_{-0.067}^{+0.068}$ & $4.486_{-0.082}^{+0.098}$ & $1.664_{-0.023}^{+0.025}$ & $1.524_{-0.035}^{+0.033}$ \\
\hline Inclination, $i\left(^{\circ}\right)$ & $27.1_{-6.1}^{+5.4}$ & $52.0_{-3.2}^{+3.6}$ & $90.0_{-19.0}^{+0.0}$ & $44.8_{-1.7}^{+1.5}$ & $56.9_{-25.1}^{+30.1 \mathrm{a}}$ & $28.2_{-25.7}^{+20.5 \mathrm{a}}$ \\
\hline Polar temperature, $T_{\mathrm{p}}(\mathrm{K})$ & $11138_{-225}^{+220}$ & $10030_{-139}^{+129}$ & $8985_{-124}^{+116}$ & $10091_{-84}^{+89}$ & $8242_{-53}^{+56}$ & $7231_{-72}^{+68}$ \\
\hline Polar position angle, $\psi\left({ }^{\circ}\right)$ & $12.1_{-54.6}^{+71.6}$ & $51.6_{-43.4}^{+42.9}$ & $154.9_{-74.8}^{+71.4}$ & $161.3_{-20.2}^{+19.8}$ & $82.5_{-15.0}^{+15.0}$ & $6.1_{-56.9}^{+75.0}$ \\
\hline Angular rotation rate, $\omega$ & $0.999_{-0.003}^{+0.001}$ & $0.964_{-0.008}^{+0.008}$ & $0.835_{-0.019}^{+0.020}$ & $0.999_{-0.001}^{+0.001}$ & $0.404_{-0.065}^{+0.051}$ & $0.530_{-0.098}^{+0.082}$ \\
\hline Polar radius, $R_{\mathrm{p}}\left(R_{\odot}\right)$ & $2.233_{-0.064}^{+0.064}$ & $1.921_{-0.044}^{+0.044}$ & $1.723_{-0.050}^{+0.050}$ & $3.037_{-0.038}^{+0.045}$ & $1.622_{-0.022}^{+0.023}$ & $1.455_{-0.032}^{+0.030}$ \\
\hline Average radius, $R_{\mathrm{avg}}\left(R_{\odot}\right)$ & $2.557_{-0.079}^{+0.077}$ & $2.147_{-0.054}^{+0.053}$ & $1.846_{-0.057}^{+0.057}$ & $3.479_{-0.045}^{+0.053}$ & $1.643_{-0.023}^{+0.024}$ & $1.488_{-0.033}^{+0.031}$ \\
\hline Average diameter, $\theta_{\text {avg }}$ (mas) & $0.932_{-0.029}^{+0.028}$ & $0.808_{-0.020}^{+0.020}$ & $0.684_{-0.021}^{+0.021}$ & $0.680_{-0.009}^{+0.010}$ & $0.408_{-0.006}^{+0.006}$ & $0.507_{-0.011}^{+0.011}$ \\
\hline Equatorial temperature, $T_{\mathrm{e}}(\mathrm{K})$ & $4724_{-1953}^{+914}$ & $6909_{-234}^{+195}$ & $7556_{-123}^{+109}$ & $3825_{-1116}^{+634}$ & $8028_{-63}^{+67}$ & $6887_{-140}^{+126}$ \\
\hline$v \sin i\left(\mathrm{~km} \mathrm{~s}^{-1}\right)$ & $171.0_{-36.2}^{+30.8}$ & $244.6_{-11.1}^{+11.6}$ & $238.6_{-13.0}^{+10.0}$ & $217.0_{-6.5}^{+5.6}$ & $85.1_{-31.6}^{+16.3}$ & $62.3_{-56.6}^{+36.8}$ \\
\hline Visibility $\chi^{2}$ & 7.646 & 2.719 & 4.498 & 0.763 & 1.083 & 1.488 \\
\hline Photometry $\chi^{2}$ & 5.798 & 3.214 & 4.021 & 2.329 & 6.313 & 5.100 \\
\hline Total $\chi^{2}$ & 13.45 & 5.933 & 8.519 & 3.092 & 7.396 & 6.588 \\
\hline
\end{tabular}

Note.

${ }^{\text {a }}$ As discussed in Section 4.4, the inclinations of 16 Lyr and 59 Dra were fixed.

calibrator's (intrinsic- $V_{\mathrm{i}}^{\mathrm{c}}$, measured- $V_{\mathrm{m}}^{\mathrm{c}}$ ):

$$
\frac{V_{\mathrm{i}}^{*}}{V_{\mathrm{i}}^{\mathrm{c}}}=\frac{V_{\mathrm{m}}^{*}}{V_{\mathrm{m}}^{\mathrm{c}}} .
$$

A common method for estimating a calibrator star's size (if it is not known from previous interferometric measurements) is by fitting a PED to measured photometry (see the Appendix). Boyajian (2009) found an average difference between angular sizes determined by PED fitting and angular sizes measured by interferometry to be $\sim 10 \%$, so a $10 \%$ error in the angular size is adopted for the calibrator stars observed for this work. Small calibrator stars are used because the smaller a star is, the less its estimated intrinsic visibility is affected by inaccuracies in its size estimate. For example, a small calibrator with a $10 \%$ error (angular diameter, $\theta=0.2 \pm 0.02$ mas) observed with the CHARA Array's longest baseline $(B=331 \mathrm{~m})$ in the $K$-band will have an estimated intrinsic visibility of $0.974 \pm 0.005$ (a $0.5 \%$ error due to the inaccuracy of an PED-determined size). A calibrator that is twice as large $(\theta=0.4 \pm 0.04$ mas $)$ and observed in the same way will have an estimated intrinsic visibility of $0.90 \pm 0.02$ (a $2.2 \%$ error due to the inaccuracy of an PED-determined size). As a rule of thumb, good calibrators are ones that are smaller than approximately half the resolution of the observation to avoid significant errors in the calibrator's visibility (van Belle \& van Belle 2005).

For this work, at least two calibrator stars were observed for each target star to help mitigate calibrator size errors. Their angular diameters are estimated by fitting PHOENIX model PEDs (Husser et al. 2013) to photometry gathered from the literature. Three of the calibrators used here (HD 177003, HD
185872, and HD 201908) had temperatures greater than the PHOENIX model grid (which goes as high as 12,000 K). For these three calibrators, the PED fits were made using ATLAS9 model PEDs (Castelli \& Kurucz 2004). Calibrator angular diameters are listed in Table 3 and range from 0.156 to 0.582 mas.

\section{FUNDAMENTAL STELLAR PROPERTIES}

\subsection{Oblate Star Model}

The limb-darkened disk model traditionally used to analyze interferometric visibilities takes neither the distended shape of rapidly rotating stars nor the gravity darkening caused by this distended shape into account. The model used here employs a Roche geometry and is based on the models used in van Belle (2012), Aufdenberg et al. (2006), and Monnier et al. (2012). In order to determine the fundamental properties of rapid rotators, the observed visibilities (Section 3 ) and broadband photometry are compared to model-predicted visibilities and photometry; the adopted photometry for each star is assembled in the Appendix. The eight input parameters for the model star are its equatorial radius $\left(R_{\mathrm{e}}\right)$, its mass $\left(M_{*}\right)$, its equatorial rotational velocity $\left(V_{\mathrm{e}}\right)$, the inclination of its polar axis relative to our line of sight $(i)$, the gravity darkening coefficient used in the model $(\beta)$, the temperature at its pole $\left(T_{\mathrm{p}}\right)$, the parallax of the observed star $\left(\pi_{\mathrm{plx}}\right)$, and the position angle of its pole $(\psi)$ with a $180^{\circ}$ ambiguity. Of these, the parallax is set by Hipparcos measurements, the gravity darkening coefficient is set by one of two possible relations (see below), and the mass is estimated from evolution models (see below). The remaining five 
Table 5

Model Results Using the ELR Gravity Darkening Law

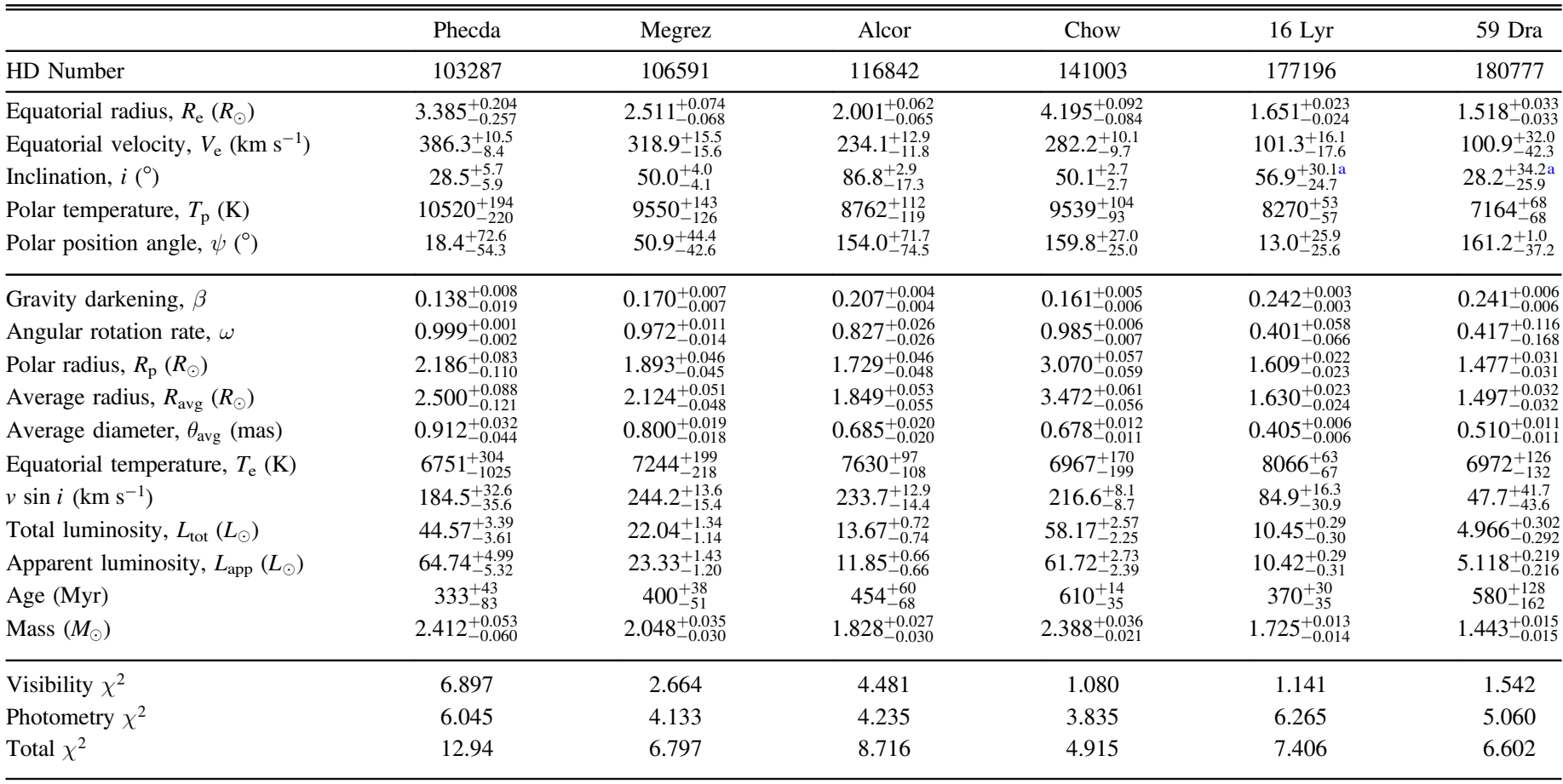

Note.

${ }^{\text {a }}$ As discussed in Section 4.4, the inclinations of 16 Lyr and 59 Dra were fixed. parameters $\left(R_{\mathrm{e}}, V_{\mathrm{e}}, i, T_{\mathrm{p}}\right.$, and $\left.\psi\right)$ are allowed to vary under the constraint that the equatorial velocity $\left(V_{\mathrm{e}}\right)$ must yield a model $v \sin i$ that is consistent with the observed $v \sin i$.

Two gravity darkening laws are incorporated here. With the canonical gravity darkening law (von Zeipel 1924a, 1924b; Claret 2000, hereafter, vZ) the stars modeled here are hot enough to have fully radiative envelopes, giving them a gravity darkening coefficient, $\beta$, of 0.25 . However, a modern gravity darkening law, tested with results from interferometric observations of rapidly rotating stars (Espinosa Lara \& Rieutord 2011, hereafter ELR) shows that $\beta$ is dependent on the angular rotation rate, $\omega$, and ranges from 0.25 for a nonrotating star $(\omega=0)$ to $\sim 0.09$ for a star rotating at its breakup velocity $(\omega=1)$.

The oblateness of a star depends not only on its rotation, but also its mass. After the best fitting free parameters are determined, the age and mass are calculated using evolution models. The mass used in the oblate star model is then updated to match the mass determined by the evolution model. The oblate star model and evolutionary model are run iteratively until neither the mass nor the free parameters change by more than $\sim 0.1 \%$ after a series of consecutive runs, corresponding to an $R_{\mathrm{e}}$ of $\sim 0.002 R_{\odot}$, a $V_{\mathrm{e}}$ of $\sim 0.2 \mathrm{~km} \mathrm{~s}^{-1}$, an $i$ of $\sim 0^{\circ} .1$, a $T_{\mathrm{p}}$ of $\sim 8 \mathrm{~K}$, and a $\psi$ of $\sim 0.4$.

To determine the ages and masses of the rapidly rotating stars in this paper the star's average radius $\left(R_{\mathrm{avg}}\right)$, total luminosity $\left(L_{\mathrm{tot}}\right)$, and equatorial velocity $\left(V_{\mathrm{e}}\right)$, as determined by the oblate star model are compared to the predictions of MESA evolutionary models (Paxton et al. 2011, 2013). These three parameters $\left(R_{\mathrm{avg}}, L_{\mathrm{tot}}\right.$, and $\left.V_{\mathrm{e}}\right)$ correspond to a star with a unique mass, age and angular velocity. The mass used by the oblate star model is set equal to the mass determined by this comparison in the iterative process described above. For this project, MESA evolution tracks ${ }^{8}$ were computed for a grid of masses and angular velocities (with resolution of $0.1 M_{\odot}$ and $10 \%$ breakup velocity, respectively) at a metallicity of $Z=0.016$ as measured by King et al. (2003) for the UMa moving group.

The stellar model is constructed by calculating the stellar intensity at each point on an oblate spheroidal grid, constructed of 51 points along the colatitudinal axis $(\vartheta)$ and 51 points along the longitudinal axis $(\varphi)$ for a total of 2601 points on the star. Then, a radius $(R(\vartheta))$ and surface gravity $(g(\vartheta)$, with radial component, $g_{\mathrm{r}}(\vartheta)$ and polar component, $g_{\vartheta}(\vartheta)$ ) are calculated for each point on the grid (van Belle 2012):

$$
\begin{gathered}
R(\vartheta)=3 \frac{R_{\mathrm{p}}}{\omega \sin \vartheta} \cos \left[\frac{\pi+\arccos (\omega \sin \vartheta)}{3}\right] \\
g(\vartheta)=\sqrt{g_{\mathrm{r}}(\vartheta)^{2}+g_{\vartheta}(\vartheta)^{2}}, \text { where } \\
g_{\mathrm{r}}(\vartheta)=\frac{-G M_{*}}{R(\vartheta)^{2}}+R(\vartheta)(\Omega \sin \vartheta)^{2} \\
g_{\vartheta}(\vartheta)=R(\vartheta) \Omega^{2} \sin \vartheta \cos \vartheta .
\end{gathered}
$$

In this prescription, $R_{\mathrm{p}}$ is the model star's polar radius:

$$
R_{\mathrm{p}}=\left[\frac{1}{R_{\mathrm{e}}}+\frac{V_{\mathrm{e}}^{2}}{2 G M_{*}}\right]^{-1},
$$

\footnotetext{
$\overline{8}$ See the related tar.gz file package for examples of MESA inlists used in this project.
} 
Table 6

Fundamental Properties of Merak (HD 95418)

\begin{tabular}{lcc}
\hline \hline & Value & Source \\
\hline Radius $\left(R_{\odot}\right)$ & $3.0210 \pm 0.0383$ & Boyajian et al. (2012) \\
Temperature $(\mathrm{K})$ & $9193 \pm 56$ & Boyajian et al. (2012) \\
Luminosity, $L_{\text {tot }}\left(L_{\odot}\right)$ & $58.46 \pm 0.47$ & Boyajian et al. (2012) \\
$v \sin i\left(\mathrm{~km} \mathrm{~s}^{-1}\right)$ & $46 \pm 2.3$ & Royer et al. (2007) \\
Inclination, $i\left(^{\circ}\right)$ & 90 & Assumed \\
Age $(\mathrm{Myr})$ & $408 \pm 6$ & This work \\
Mass $\left(M_{\odot}\right)$ & $2.509 \pm 0.005$ & This work \\
\hline
\end{tabular}

$\omega$ is the angular velocity of the star relative to its critical velocity, $\Omega_{\text {crit }}$ :

$$
\begin{aligned}
\omega & =\sqrt{\frac{27}{4} w_{0}\left(1-w_{0}\right)^{2}} \\
w_{0} & =\frac{V_{\mathrm{e}}^{2} R_{\mathrm{p}}}{2 G M_{*}}
\end{aligned}
$$

and $\Omega$ is the angular velocity of the star in radians per second:

$$
\Omega=\omega \Omega_{\text {crit }}=\omega\left(\frac{8}{27} \frac{G M_{*}}{R_{\mathrm{p}}^{3}}\right)^{1 / 2} .
$$

This allows the gravity dependent surface temperature $(T(\vartheta))$ to be calculated at each point on the grid:

$$
T=T_{\mathrm{p}}\left(\frac{g(\vartheta)}{g_{\mathrm{p}}}\right)^{\beta}
$$

where $g_{\mathrm{p}}$ is the surface gravity at the model star's pole:

$$
g_{\mathrm{p}}=\frac{G M_{*}}{R_{\mathrm{p}}^{2}} .
$$

A grid $^{9}$ of PHOENIX atmosphere models (Husser et al. 2013) are interpolated to determine the intensity spectrum at each point on the stellar model surface grid based on the temperature and surface gravity of those points.

Model photometry is calculated by integrating the 2601 intensity spectra that cover the star to compute the flux spectrum of the star, $F_{\lambda}$ :

$$
F_{\lambda}=\int_{\vartheta=0}^{\pi} \int_{\varphi=0}^{2 \pi} I_{\lambda}(\vartheta, \varphi) \theta_{\mathrm{R}}^{2}(\vartheta) \sin (\vartheta) \mu(\vartheta, \varphi) d \varphi d \vartheta
$$

$I_{\lambda}(\vartheta, \varphi)$ is the intensity spectrum given by the PHOENIX model. $\theta_{\mathrm{R}}(\vartheta)$ is the angular radius of the model star as a function of colatitude. $\mu(\vartheta, \varphi)$ is the cosine of the angle between the observer and the normal of the star:

$$
\begin{aligned}
\mu(\vartheta, \varphi)= & \frac{1}{g(\vartheta)}\left[-g_{\mathrm{r}}(\vartheta)(\sin (\vartheta) \sin (i) \cos (\varphi)\right. \\
& +\cos (\vartheta) \cos (i)) \\
& \left.-g_{\vartheta}(\vartheta)(\sin (i) \cos (\varphi) \cos (\vartheta)-\sin (\vartheta) \cos (i))\right]
\end{aligned}
$$

\footnotetext{
$\overline{9}$ Grid step sizes are 0.5 in $\log g$ and $200 \mathrm{~K}$ in $T_{\text {eff }}$.
}

Note that $I_{\lambda}(\vartheta, \varphi)=0$ for $\mu<0$ (i.e., only light directed at the observer is included in the integration). The resulting flux spectrum is convolved with the appropriate bandpass filter to compute the specific flux from which the photometry is calculated.

The bolometric flux is simply $F_{\text {bol }}=\int F_{\lambda} d \lambda$ and the apparent luminosity is then $L_{\text {app }}=4 \pi F_{\text {bol }} d^{2}$. The total luminosity, $L_{\text {tot }}$, is calculated by determining $J_{\lambda}$, the specific irradiance on each point

$$
J_{\lambda}\left(T_{\text {eff }}, g\right)=\int_{\mu=0}^{1} I_{\lambda}\left(T_{\text {eff }}, g, \mu\right) \mu d \mu
$$

integrating over all wavelengths:

$$
J_{\mathrm{bol}}(\vartheta)=2 \pi \int_{\lambda} J_{\lambda}\left(T_{\mathrm{eff}}, g\right) d \lambda
$$

and integrating over the model star's surface:

$$
L_{\mathrm{tot}}=2 \pi \int_{\vartheta=0}^{\pi} J_{\mathrm{bol}}(\vartheta) R^{2}(\vartheta) \sin (\vartheta) d \vartheta .
$$

Model visibilities are calculated by first creating an image of the model star in the bandpass of the observations. For example, if the visibilities are observed in $H$-band, the intensity spectra at the different points in the image are convolved with an $H$-band filter. A 2D fast Fourier transform (FFT) is taken of that synthetic image. This image is $4900 \times 4900$ pixels with $\sim 1000$ of those pixels (in the center of the image) being made up of synthetic starlight. This distribution is designed to produce an image that is high enough resolution to detect the oblateness and for the FFT to extract accurate visibilities. The model squared visibility is the complex square of that transform at the observed $u$ and $v$ spatial frequencies and the model visibilities are the square root of that quantity.

The above prescription yields visibilities and photometry based on a model star that can be tuned to match the observations. A random search algorithm is employed to find the set of free-parameters $\left(R_{\mathrm{e}}, V_{\mathrm{e}}, i, T_{\mathrm{p}}\right.$, and $\left.\psi\right)$ that minimizes the difference between observed and model predictions. For each set of input parameters, a reduced $\chi^{2}$ goodness-of-fit metric is calculated with five degrees of freedom for both the visibilities and the photometry. The final $\chi^{2}$ (hereafter, $\chi_{\text {tot }}^{2}$ ) is then calculated by adding the $\chi^{2}$ values of the visibility data and those of the photometry, assuming equal weight for the two. The search algorithm randomly selects a set of parameters within a given window of parameter space. The initial window size for the set of parameters, $\left(R_{\mathrm{e}}, V_{\mathrm{e}}, i, T_{\mathrm{p}}, \psi\right)$ is $\left( \pm 0.5 R_{\odot}\right.$, $\left.\pm \frac{\sigma_{v} \sin i}{\sin (i)} \mathrm{km} \mathrm{s}^{-1}, \pm 20^{\circ}, \pm 500 \mathrm{~K}, \pm 30^{\circ}\right)$, and this search area is decreased over multiple steps, eventually reaching $\left( \pm 0.01 R_{\odot}\right.$, $\pm 1 \mathrm{~km} \mathrm{~s}^{-1}, \pm 1^{\circ}, \pm 1 \mathrm{~K}, \pm 1^{\circ}$ ). This window is initially centered on the initial guess parameters, but it is re-centered whenever a model with a smaller $\chi_{\text {tot }}^{2}$ is calculated. The best fitting model is determined by minimizing the $\chi_{\text {tot }}^{2}$ after multiple iterations. The error for each of the five free-parameters is found by first varying them independently until the $\chi_{\text {tot }}^{2}$ increases by 1 after first scaling the $\chi_{\text {tot }}^{2}$ such that the minimum $\chi_{\text {tot }}^{2}=1$.

Due to the large scatter in the broad-band photometric measurements relative to their error, the best fitting model finds an unscaled $\chi_{\text {tot }}^{2}$ of $\gtrsim 100$ (dominated by the photometric $\chi^{2}$ ) when adopting the published errors for the photometry measurements, the mean and median of which are 0.016 and 

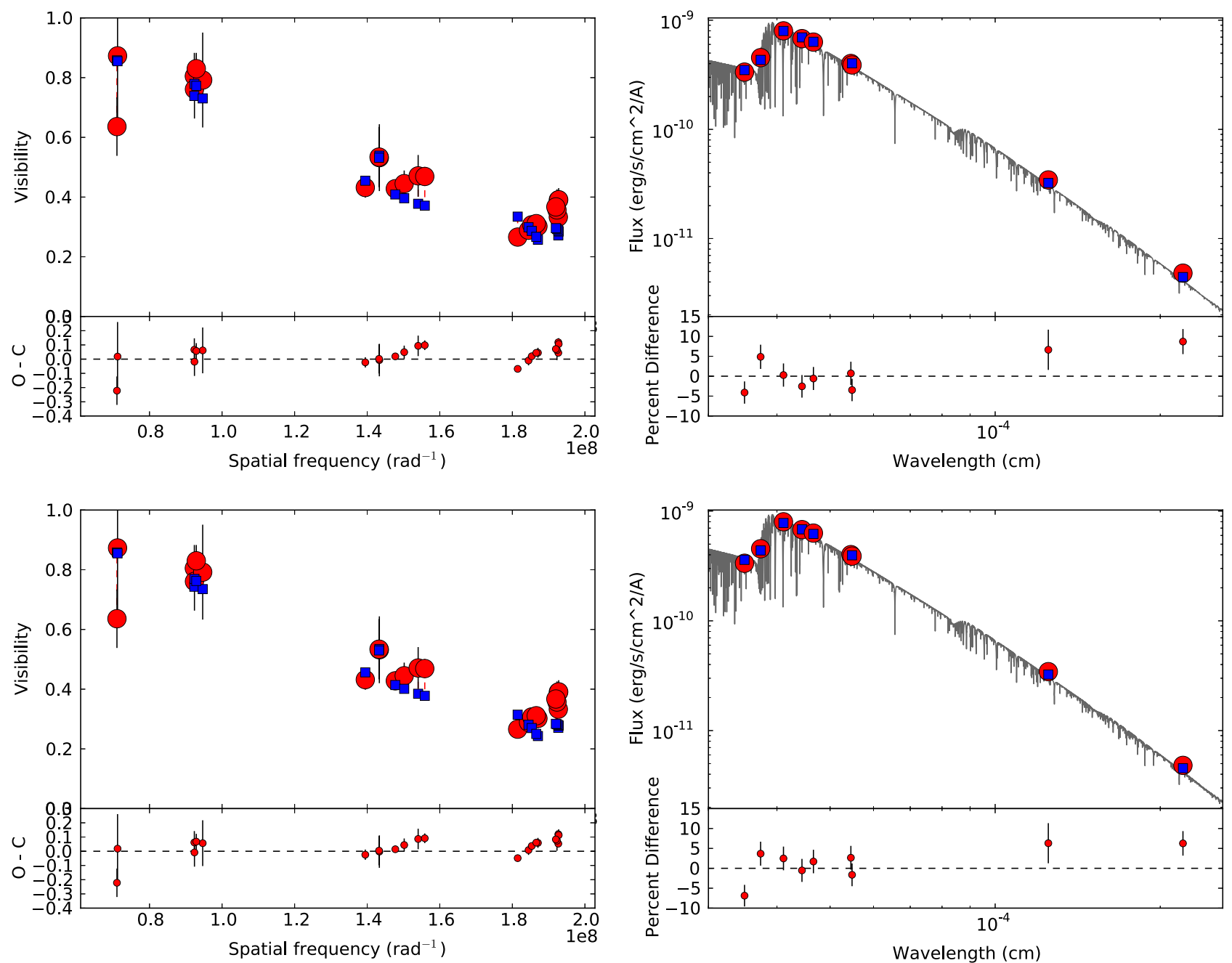

Figure 4. Top left-visibility measurements (red circles) for Phecda (HD 103287) are compared to the best fit model visibilities (blue squares) assuming the ELR prescription for gravity darkening. Dashed lines connect individual model and measured values and solid lines are the error bars. Top right-photometric measurements (red circles) for Phecda (HD 103287) are compared to the best fit model photometry (blue squares) assuming the ELR prescription for gravity darkening. The spectral energy distribution from which the PED is calculated is plotted in gray for comparison. Bottom left—same as top left, but for the vZ gravity darkening law. Bottom right—-same as top right, but for the vZ gravity darkening law.

$0.011 \mathrm{mag}$, respectively. More importantly, few of the photometric measurements overlapped with the model PED which could indicate underestimates of the photometric error, inaccuracies of the synthetic spectral energy distribution, incorrect filter profiles or zero-points, etc. To account for this, photometric errors of $0.03 \mathrm{mag}$ were adopted for all photometric values which had an error less than $0.03 \mathrm{mag}$. With these adopted photometric errors, all of the best fitting models had an unscaled $\chi_{\text {tot }}^{2}$ of $<15$.

To determine the errors in the age and mass, the age and mass are calculated for the ten points which represent the $1 \sigma$ errors of the five parameters in the oblate star model (i.e., $\left[R_{\mathrm{e}} \pm \sigma_{R_{\mathrm{e}}}, V_{\mathrm{e}}, i, T_{\mathrm{p}}, \psi\right],\left[R_{\mathrm{e}}, V_{\mathrm{e}} \pm \sigma_{V_{\mathrm{e}}}, i, T_{\mathrm{p}}, \psi\right]$, etc.). The lowest and highest values that come from this procedure represent the lower and upper bounds of the statistical errors presented here. We note that this method does not take into account any correlations that may be present between the free parameters. The final best fitting parameters and their errors for the $\mathrm{vZ}$ and ELR gravity darkening laws can be found in Tables 4 and 5, respectively. Figures 4-9 illustrate the best fitting model visibilities and photometry, as constrained by the observations, for both gravity darkening prescriptions.

\subsection{Initial Model Parameters}

The $\chi^{2}$ minimization technique that is used to determine the best-fitting model (see Section 4.1) is especially sensitive to the initial guess given for the star's inclination. To account for this, for each star, the model is run a number of times using various fixed inclinations. The inclinations chosen range from $90^{\circ}$ (edge-on) down to an inclination that would have the model star rotating at breakup velocity given its $v \sin i$. The bestfitting set of parameters of these fixed-inclination models is chosen as the set of input parameters for the process described in Section 4.1.

The initial guess value for $M_{*}$ that is supplied for the model runs at fixed inclinations is determined based on the star's spectral type and the spectral type-mass relations found in Cox (2000). The initial guess values for $R_{\mathrm{e}}$ and $T_{\mathrm{p}}$ are based on the angular diameters and effective temperatures listed in the JMMC Stellar Diameter Catalog (Lafrasse et al. 2010) for each star. The initial value for $\psi$ is determined by fitting a uniform ellipse to the visibilities in the cases where multiple baseline orientations have been used or is set to $0^{\circ}$ in the cases where they have not. 

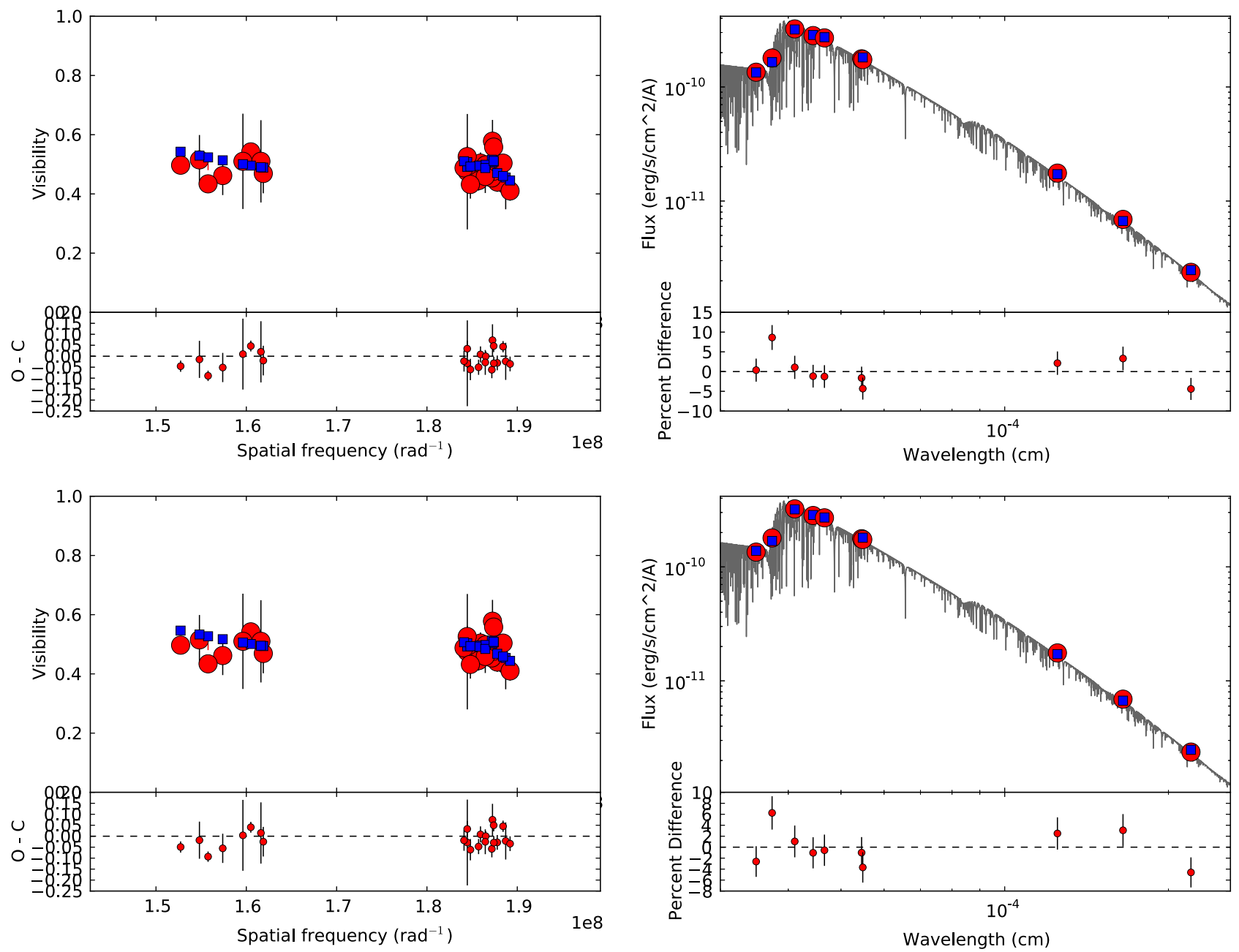

Figure 5. Same as Figure 4, but for Megrez (HD 106591).

\subsection{Merak}

The apparent slow rotator, Merak (HD 95418), was observed using the Classic beam combiner on the CHARA Array previously by Boyajian et al. (2012). We have taken the radius and luminosity determined by that study as well as its $v \sin i$ to determine its age and mass using the MESA evolution model using a similar process described in Section 4.1, but without any iteration. Because of this, we do not determine the inclination of this star nor its equatorial velocity. We assume an edge-on inclination of $90^{\circ}$. The results are compiled in Table 6.

\section{4. $16 \mathrm{Lyr}$ and 59 Dra}

When running the model described above, the two stream stars, 16 Lyr and 59 Dra, both yield best fitting values for $R_{\mathrm{avg}}$ and $L_{\text {tot }}$ that correspond to unphysical positions below the zeroage main sequence for their respective best fit values for $V_{\mathrm{e}}$. One way to reconcile this discrepancy would be for the stars to have a metallicity of $Z \lesssim 0.013(\sim 0.1 \mathrm{dex}$ lower than the moving group). We are cautious against advocating for this interpretation since, as discussed in Section 2, we have insufficient baseline orientations to fully measure the oblateness and gravity darkening in these cases. We note that the best fitting values for $V_{\mathrm{e}}$ for both $16 \mathrm{Lyr}$ and 59 Dra are sufficiently large that they shift the zero-age main sequence above the best fitting values for $R_{\mathrm{avg}}$ and $L_{\mathrm{tot}}$. If these $V_{\mathrm{e}}$ values are too large, this could explain the unphysical $R_{\mathrm{avg}}$ and $L_{\mathrm{tot}}$ without changing the metallicity. Figure 1 illustrates how the zeroage main sequence is raised by rapid rotation. With this in mind, we run the model for these two stars constraining the equatorial velocity to be within the more modest range of 94-202 $\mathrm{km} \mathrm{s}^{-1}$ for each star. This range corresponds to the dispersion about the maximum of the probability distribution of equatorial rotation velocities for late-type A-stars as determined by Zorec \& Royer (2012). We make this constraint by fixing the stars' inclinations such that $i=\arcsin \left(\frac{v \sin i}{E\left[V_{\mathrm{e}}\right]}\right)$ where $E\left[V_{\mathrm{e}}\right]$ is the maximum of the aforementioned probability distribution. This corresponds to inclinations of $\sim 57^{\circ}$ and $\sim 28^{\circ}$ for $16 \mathrm{Lyr}$ and 59 Dra, respectively.

\section{MODEL RESULTS}

\subsection{Photospheric Properties}

Using the procedure described in Section 4.1, the best fitting models for all six of the stars observed show $\chi_{\text {tot }}^{2}$ values ranging from 3.1 to 13.4 . The model fitting using the $\mathrm{vZ}$ gravity darkening law yield a high inclination $\left(i>70^{\circ}\right)$ for one star (Alcor), moderate inclinations $\left(40^{\circ}>i<70^{\circ}\right)$ for two stars (Megrez and Chow), and a low inclination $\left(i<40^{\circ}\right)$ for one star (Phecda); both 16 Lyr and 59 Dra have fixed inclinations 

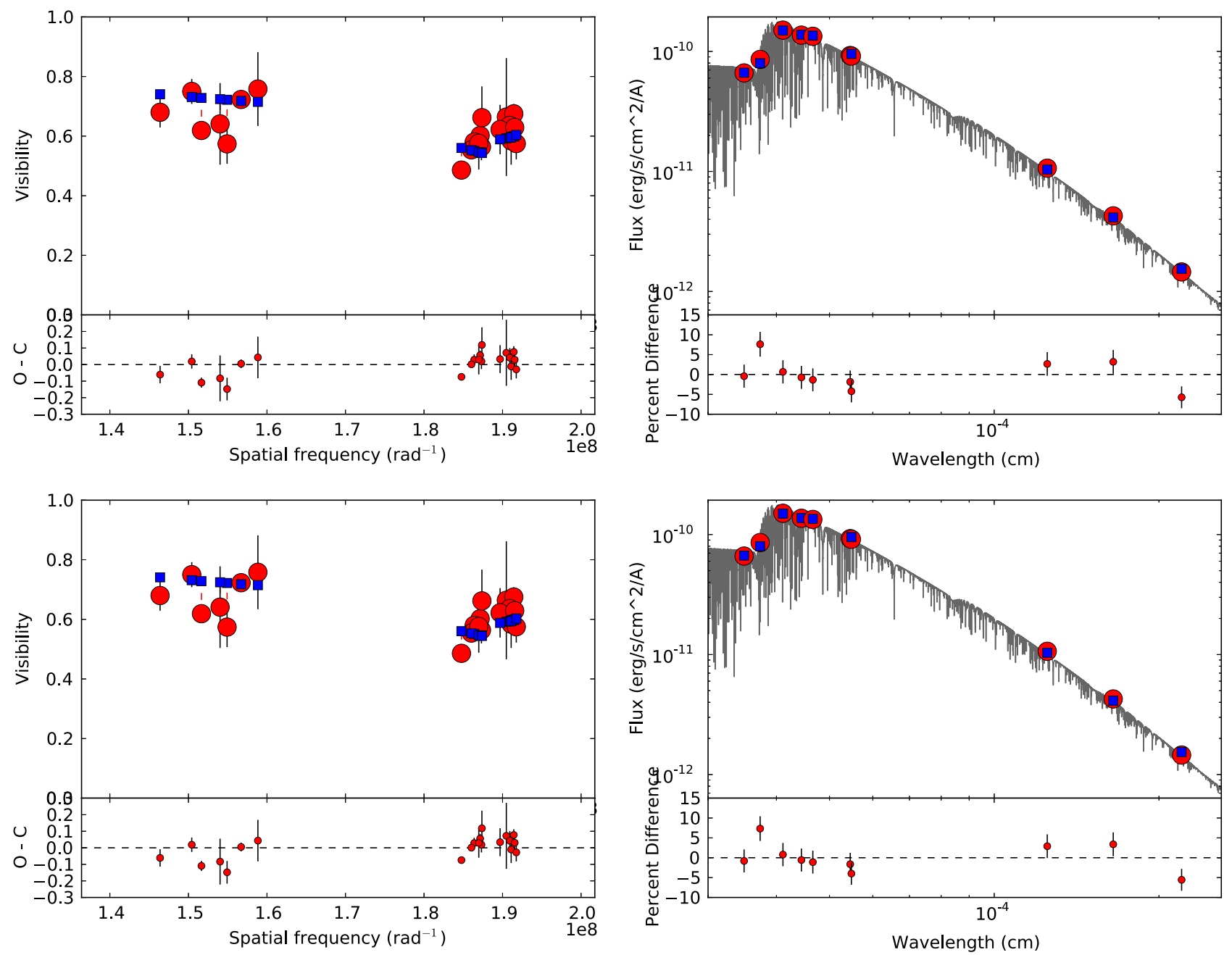

Figure 6. Same as Figure 4, but for Alcor (HD 116842).

(see Section 4.4). These results also show an oblateness, $\rho=\left(R_{\mathrm{e}}-R_{\mathrm{p}}\right) / R_{\mathrm{p}}$ that ranges from $3 \%$ to $54 \%$ with an average of $26 \%$ and temperature differences across the photosphere, $\Delta T=T_{\mathrm{p}}-T_{\mathrm{e}}$ that range from 214 to $6414 \mathrm{~K}$ with an average of $2965 \mathrm{~K}$. The same analysis done using the ELR gravity darkening law also yields a high inclinations for Alcor, moderate inclinations for four Megrez and Chow, and a low inclination for Phecda. These results show an oblateness range of $3 \%$ to $55 \%$ with an average of $24 \%$ and temperature differences across the photosphere that range from 192 to $3769 \mathrm{~K}$ with an average of $1696 \mathrm{~K}$. The smaller mean temperature gradient seen with the ELR law is because that law yields a smaller gravity darkening coefficient, $\beta$, which lessens the effect the local surface gravity has on the local temperature. Using the vZ law, $\beta$ is 0.25 for all four observed rapid rotators. The ELR law has $\beta$ ranging from 0.138 to 0.242 .

\subsection{Masses and Ages}

The masses calculated by the procedures discussed in Section 4 range from 1.447 to $2.509 M_{\odot}$ for all seven stars in the sample using the vZ gravity darkening law and 1.443-2.509 $M_{\odot}$ using the ELR law (Figure 10). The mass estimates for the individual stars are consistent between the two laws within their 1\%-3\% uncertainties with the exception of Chow, whose mass is $2.333_{-0.015}^{+0.015} M_{\odot}$ using the vZ law or
$2.388_{-0.021}^{+0.036} M_{\odot}$ using the ELR law. The ages calculated by the procedures described above range from 401 to $659 \mathrm{Myr}$ for all seven stars in the sample using the $\mathrm{vZ}$ gravity darkening law and 333-610 Myr using the ELR law. With the exception of the star Chow, these age estimates are consistent with being coeval using either the vZ and ELR laws, despite their larger uncertainties, that range from $2 \%$ to $41 \%$ and with a mean and median uncertainty of $14 \%$ and $12 \%$, respectively. It is worth noting that the uncertainty in the age is partially dependent on the mass because the radius, luminosity, and temperature of more massive stars evolve more rapidly, thus allowing for a more precise determination of the age because fixed uncertainties in these parameters will correspond to a smaller percent error in the age. We caution that these uncertainties are only statistical. Systematic uncertainties (such as those in gravity darkening and metallicity) can lead to more substantial errors. Only Chow shows a disparity in its age estimates between the two gravity darkening laws. Chow's age is determined to be $659_{-10}^{+11} \mathrm{Myr}$ when using the vZ law or $610_{-35}^{+14}$ Myr when using the ELR law. The final ages and masses for the are presented in Table 7.

\subsection{Comparison with Other Evolution Models}

In order to test the accuracy of the MESA evolution models and to begin to address some of the systematic errors that may 

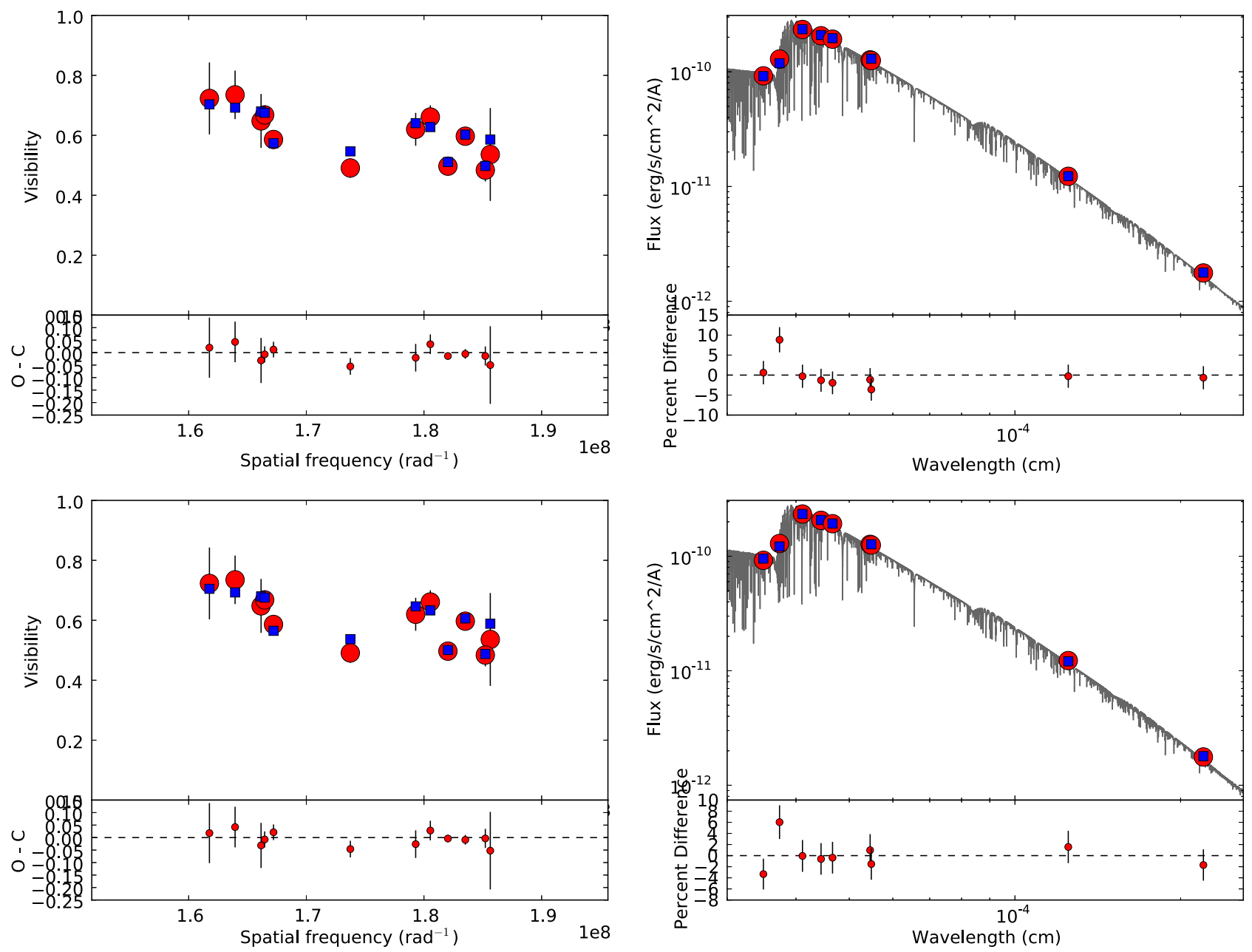

Figure 7. Same as Figure 4, but for Chow (HD 141003).

be introduced by them, we compare the results from one of the stars in our sample across four different evolution models: the MESA models; the Geneva models (Georgy et al. 2013), which do take rotation into account; the Padova models (Girardi et al. 2002), which do not account for rotation; and the MESA models again, but without accounting for rotation. We use the total luminosity, average radius, and equatorial rotation velocity determined for Alcor (HD 116842) ${ }^{10}$ as our point of comparison between the four models. We chose Alcor for this comparison because it is the only rapidly rotating nucleus member whose rotation speed is less than the maximum predicted by the Geneva models, which are restricted to values of $\omega$ of $\lesssim 0.9$ for the masses and ages in question. The results are listed in Table 8.

The absolute ages agree extremely well between the two rotating models, with a percentage difference of only $0.5 \%$ $(0.02-\sigma)$. The determined stellar masses also show good agreement, with a percentage difference of $3.1 \%(1.4-\sigma)$. The ages determined by the non-rotating models also agree with each other extremely well with a percentage difference of $0.9 \%$ $(0.07-\sigma)$, but as expected, they are systematically older than those determined using the models that account for rotation. The masses determined by the non-rotating models also show

${ }^{10}$ Using the $\mathrm{vZ}$ gravity darkening law. good agreement with each other with a percentage difference of $2.1 \%(1.0-\sigma)$.

\subsection{A New Age Estimate for the UMa Moving Group}

The mean age, uncertainty in the mean, and standard deviation of the 7 Ursa Major moving group A-stars presented here are 451,32 , and $86 \mathrm{Myr}$ when using the vZ gravity darkening law and 451, 37, and $98 \mathrm{Myr}$ when using the ELR law. These large standard deviations are due in large part to the relatively old age we estimate for Chow $\left(659_{-10}^{+11} \mathrm{Myr}\right.$ for the $\mathrm{vZ}$ law or $610_{-35}^{+14}$ Myr for the ELR law).

The discrepant age for Chow questions its association with the moving group. Of the seven stars studied here, Chow is one of two stars considered to be a "probable member" by King et al. (2003); the other five are classified as members. As assembled in King et al. (2003), its space motion is consistent with that of nucleus members, despite being $23 \mathrm{pc}$ further away (Table 2). Since we cannot confidently exclude Chow as a member, we give statistics both with and without it. If Chow is excluded, we determine a mean age and standard deviation for the six remaining stars to be $416 \pm 11 \mathrm{Myr}$ when using the vZ law and $424 \pm 79$ Myr when using the ELR law.

A primary goal of this work is to use the ensemble of stellar ages to provide a new, independent age estimate for the Ursa Major moving group. The distributions of individual ages in 

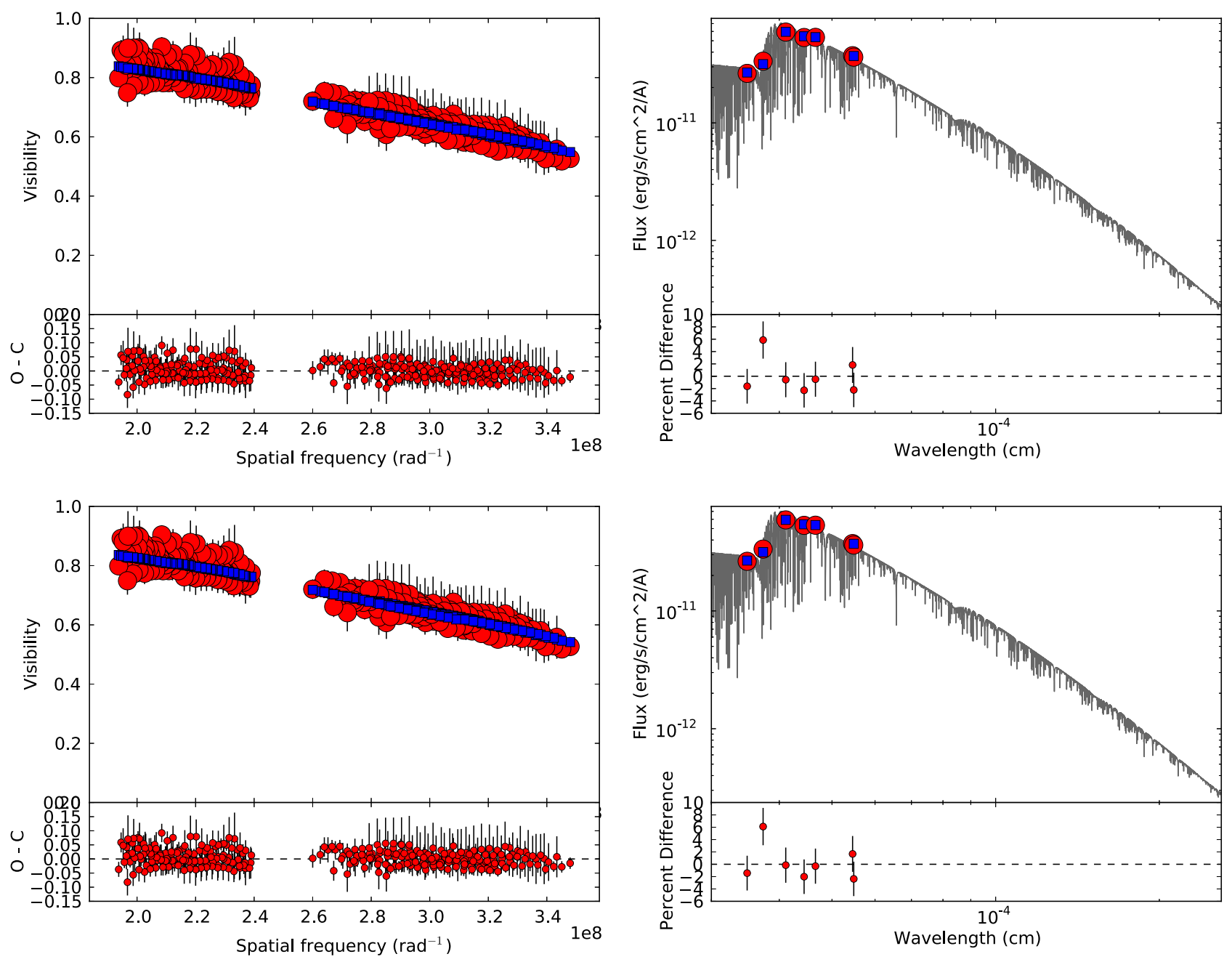

Figure 8. Same as Figure 4, but for 16 Lyr (HD 177196).

Figure 10, however, illustrate the challenge of doing this robustly as the determined ages contain systematic uncertainties (e.g., gravity darkening), a broad range of statistical uncertainties (that can bias weighted values), and possible nonmembers (e.g., Chow). Beers et al. (1990) discuss a variety of statistically robust techniques for computing the central location ("mean") and scale ("dispersion") of small samples that are potentially contaminated with outliers or that have an unknown underlying distribution. Following their recommendations, we choose to compute a median for the central location of the age and use a technique known as the "gapper" to estimate the dispersion in our sample (see Wainer \& Thissen 1976). A median is better in this case because it is influenced much less by any individual point than a mean would be. A median is also preferred over a weighted mean for this sample because of the broad range of uncertainties that may not account for all systematic uncertainties. The gapper method is based on the size of the intervals (or "gaps") in an ordered set of measurements with the "gaps" near the median being weighted more heavily. The gapper is normalized such that it is equivalent to a standard deviation. The median age and gapper scale $\left(\sigma_{\mathrm{g}}\right)$ of the seven A-stars presented here are $415 \pm$ 71 Myr when using the vZ law and $408 \pm 110$ when using the ELR law.

Since the gapper scale is intended to approximate the standard deviation for a Gaussian distribution, we use it to define an uncertainty in the median as $\frac{\sigma_{\mathrm{g}}}{\sqrt{n}}$, following standard convention. The median, gapper scale, uncertainty in the median, mean, and standard deviation are presented in Table 9 for three distinct subsamples of the seven stars observed. The first of these subsamples is the four nucleus stars (Merak, Phecda, Megrez, and Alcor) which are considered bona fide members of the moving group, and so are of greater interest in determining the age of the group. We find a median age and gapper scale of $415 \pm 6 \mathrm{Myr}$ and $404 \pm 55 \mathrm{Myr}$ for the $\mathrm{vZ}$ and ELR laws, respectively. The second of these samples is the full sample of seven stars with an age of $415 \pm 71 \mathrm{Myr}$ (vZ) and $408 \pm 110 \mathrm{Myr}$ (ELR). The final sample is the full sample excluding Chow which, due to its estimated old age, may be an interloper. Without Chow, we find a vZ age of $415 \pm 13 \mathrm{Myr}$ and an ELR age of $404 \pm 88 \mathrm{Myr}$.

As discussed in Section 5.1, the model results using the two gravity darkening laws show no considerable difference for individual stars. The vZ law, as illustrated in Figures 10-11, does yield more consistent age estimates $\left(\sigma_{\mathrm{g}}=13 \mathrm{Myr}\right)$ among the observed stars (excluding Chow) than the ELR law does $\left(\sigma_{\mathrm{g}}\right.$ $=88 \mathrm{Myr}$ ). However, given that many of the uncertainties in the individual measurements are as large or larger than the dispersion in the age estimates, we consider that this may be a statistical anomaly. Because of this, we hesitate to favor one law over the other. 

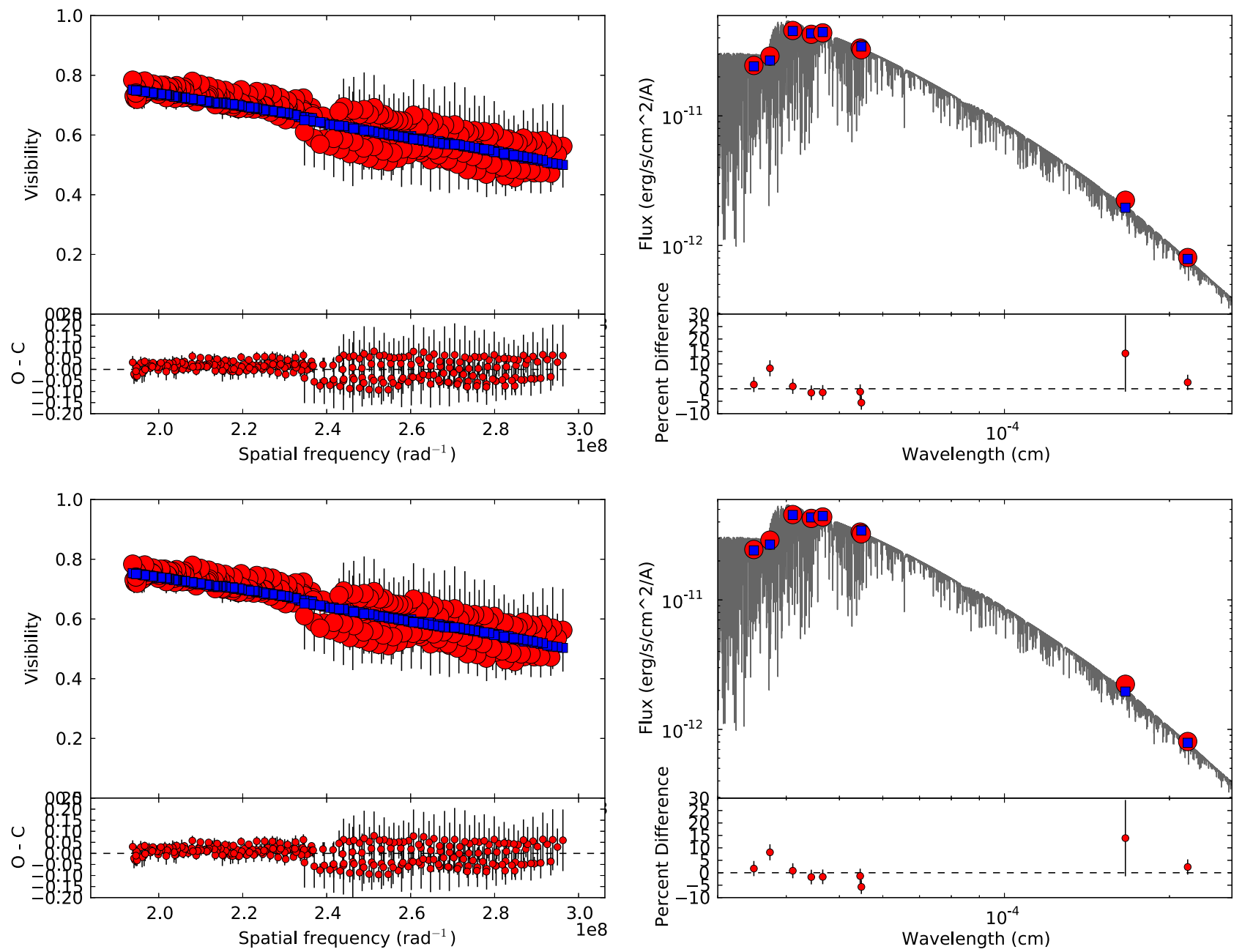

Figure 9. Same as Figure 4, but for 59 Dra (HD 180777).

Table 7

Ages and Masses for Individual Stars

\begin{tabular}{lcccc}
\hline \hline \multirow{2}{*}{$\begin{array}{l}\text { Star } \\
\text { Name }\end{array}$} & \multicolumn{2}{c}{ Mass $\left(M_{\odot}\right)$} & \multicolumn{2}{c}{ Age (Myr) } \\
& & ELR Law & vZ Law & ELR Law \\
\hline Merak & $2.509 \pm 0.005$ & \multicolumn{2}{c}{$408 \pm 6$} \\
Phecda & $2.348_{-0.060}^{+0.055}$ & $2.412_{-0.060}^{+0.053}$ & $415_{-61}^{+53}$ & $333_{-83}^{+43}$ \\
Megrez & $2.062_{-0.033}^{+0.030}$ & $2.048_{-0.030}^{+0.035}$ & $414_{-43}^{+35}$ & $400_{-51}^{+38}$ \\
Alcor & $1.842_{-0.031}^{+0.027}$ & $1.828_{-0.030}^{+0.027}$ & $422_{-75}^{+67}$ & $454_{-68}^{+60}$ \\
Chow & $2.333_{-0.015}^{+0.015}$ & $2.388_{-0.021}^{+0.036}$ & $659_{-10}^{+11}$ & $610_{-35}^{+14}$ \\
16 Lyr & $1.722_{-0.013}^{+0.013}$ & $1.725_{-0.014}^{+0.013}$ & $401_{-32}^{+31}$ & $370_{-35}^{+30}$ \\
59 Dra & $1.447_{-0.015}^{+0.014}$ & $1.443_{-0.015}^{+0.015}$ & $436_{-203}^{+156}$ & $580_{-162}^{+128}$ \\
\hline
\end{tabular}

To estimate the age of the moving group, we combine the following into one set of age estimates: the age of Merak determined using the method described in Section 4.3; the ages of Phecda, Megrez, Alcor, 16 Lyr, and 59 Dra as determined using the vZ law; and the ages of those same five stars as determined using the ELR law. This combined set of ages allow us to sample what our technique can achieve by accounting for the full spread in ages we estimate using two gravity darkening laws. With this combined set, we find the median age and uncertainty in the median of the moving group to be $414 \pm 23 \mathrm{Myr}$.
Table 8

Comparing Evolution Models

\begin{tabular}{ll}
\hline \hline \multicolumn{2}{c}{ Fundamental Parameters for Alcor (HD 116842) } \\
\hline Average radius $\left(R_{\odot}\right)$ & $1.846_{-0.057}^{+0.057}$ \\
Total luminosity, $L_{\text {tot }}\left(L_{\odot}\right)$ & $13.98_{-0.75}^{+0.75}$ \\
Equatorial velocity $\left(\mathrm{km} \mathrm{s}^{-1}\right)$ & $238.6_{-9.2}^{+10.0}$ \\
\hline
\end{tabular}

\begin{tabular}{lll}
\hline & MESA (with Rotation) \\
\hline Age (Myr) & $422_{-75}^{+67}$ \\
Mass $\left(M_{\odot}\right)$ & $1.842_{-0.031}^{+0.027}$ \\
\hline & Geneva (with Rotation) \\
\hline Age (Myr) & \\
Mass $\left(M_{\odot}\right)$ & & $424_{-75}^{+69}$ \\
& & $1.899_{-0.029}^{+0.026}$
\end{tabular}

\begin{tabular}{lll}
\hline & MESA (without Rotation) \\
\hline Age $(\mathrm{Myr})$ & & $575_{-41}^{+45}$ \\
Mass $\left(M_{\odot}\right)$ & $1.817_{-0.027}^{+0.027}$ \\
\hline & & \\
\hline Age $(\mathrm{Myr})$ & Padova (without Rotation) & $580_{-56}^{+54}$ \\
Mass $\left(M_{\odot}\right)$ & $1.855_{-0.029}^{+0.027}$ \\
\hline
\end{tabular}



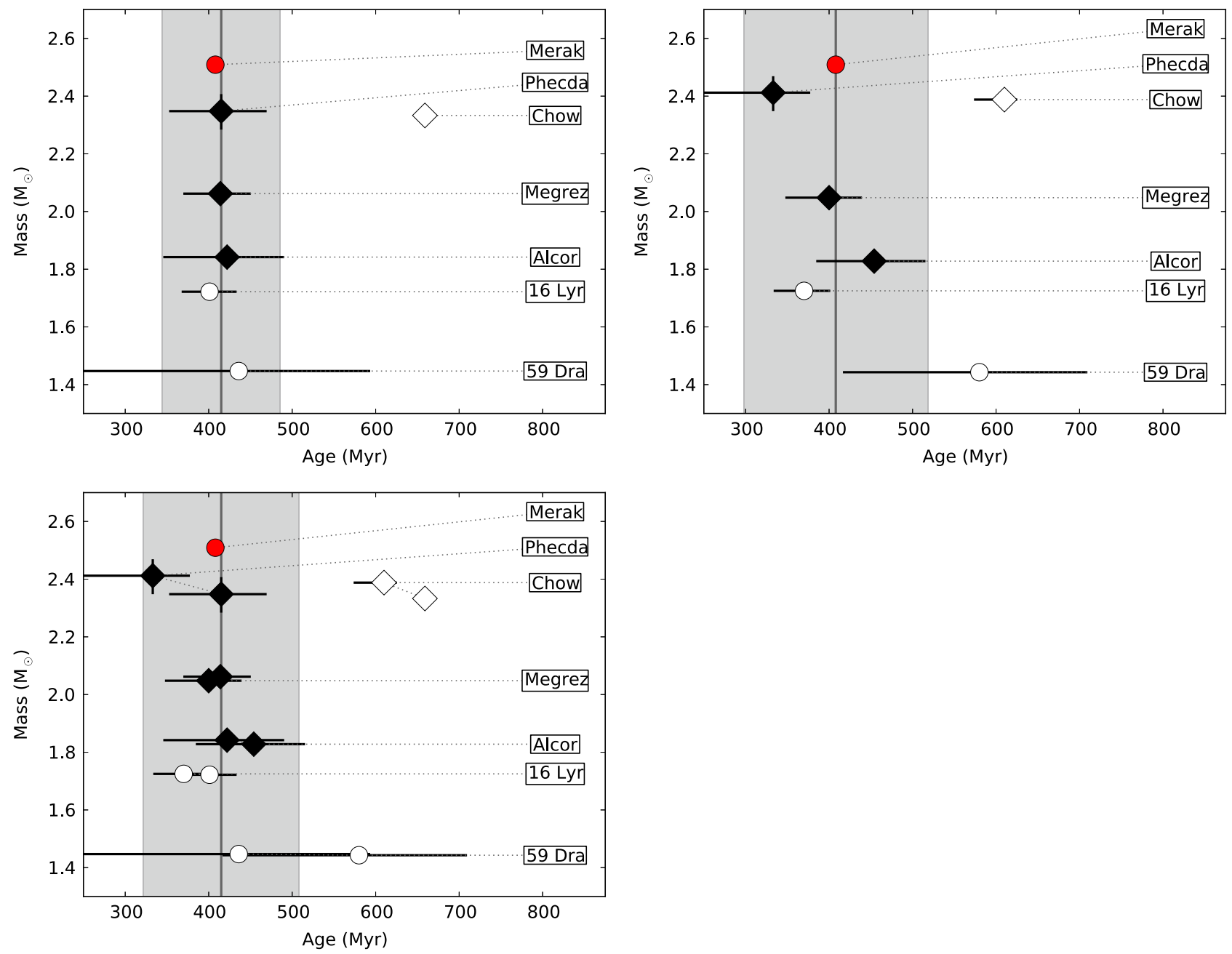

Figure 10. Distribution of stellar masses vs. age for seven stars in the Ursa Major moving group as determined using the vZ gravity darkening law (a), ELR law (b), and both (c) with the model described in Section 4.1. The circles are slowly rotating stars $\left(V_{\mathrm{e}}<170 \mathrm{~km} \mathrm{~s}^{-1}\right)$ and the diamonds are rapidly rotating $\left(V_{\mathrm{e}}>170 \mathrm{~km} \mathrm{~s} \mathrm{~s}^{-1}\right)$. The black points are nucleus members and the white points are stream members. The red point shows the mass and age of the nucleus member, Merak, that was previously observed by Boyajian et al. (2012) and is discussed in Section 4.3. In some cases, the size of the statistical error bar is smaller than the size of the symbol. The dark vertical lines represent the median in the ages and the shaded regions represent the gapper scale (the standard deviation equivalent discussed in Section 5.4). The dotted lines in (c) connect the age and mass estimates from the two different laws.

\subsection{Model Precision in the Age Estimate for Isolated A-Stars}

Under the assumption that these stars are the same age, the resulting coeval ages provide validation of not only the model presented here, but also the MESA evolution model and the physics assumed therein. The dispersion of ages can be used to quantify the precision of this technique when applied to isolated adolescent-age A-stars. Only three stars (Phecda, Megrez, and Alcor) of the observed seven are both considered bona fide nucleus members of the moving group and were fully modeled by the technique presented in Section 4.1. The median and gapper scale of their six age estimates (an age estimate using the vZ law and one using the ELR law for each star) is $415 \pm 40 \mathrm{Myr}$. We use this scale value to determine a precision in our model of $\sim 10 \%$ for stars with masses ranging from $\sim 1.8$ to $2.4 M_{\odot}$ and at a few hundred Myr age. Therefore when using this technique on field A-stars we expect an overall uncertainty of $10 \%$ in the age estimates.

\section{SUMMARY}

We present new interferometric observations for six A-type stars in the Ursa Major moving group and new age and mass estimates for these six plus one that was observed previously. All observations were made using the Classic, CLIMB, and PAVO beam combiners on the CHARA Array. Four of the observed stars are known to be rapidly rotating with $v \sin i \gtrsim$ $170 \mathrm{~km} \mathrm{~s}^{-1}$ causing them to be measurably oblate.

To properly account for this rapid rotation, a model was constructed with a Roche geometry based on eight parameters: $R_{\mathrm{e}}, M_{*}, V_{\mathrm{e}}, i, \beta, T_{\mathrm{p}}, \pi_{\mathrm{plx}}$, and $\psi$. Visibilities and photometry were calculated for each star using model-generated images and PEDs, and then compared to measured visibilities and photometry. Five of the model parameters $\left(R_{\mathrm{e}}, V_{\mathrm{e}}, i, T_{\mathrm{p}}\right.$, and $\psi$ ) were allowed to vary, with $V_{\mathrm{e}}$ constrained by $i$ and the measured $v \sin i$. Age and mass estimates were made for each of the stars in this sample by comparing their modeled average radius, luminosity, and equatorial velocity of to those parameters determined by MESA evolution models. The mass determined by the MESA model was then used in the Roche model and this process was repeated until the models converged.

Two different gravity darkening laws were studied. Neither law was favored by the interferometric and photometric data, nor was either law favored by the final age estimates. The dispersion in the age estimates was significantly smaller for the 
Table 9

Age Estimates and Uncertainties (in Myr) for Various Subsets

\begin{tabular}{|c|c|c|c|c|c|c|c|c|c|}
\hline & \multirow[b]{2}{*}{$n$} & \multirow[b]{2}{*}{$n^{*}$} & \multicolumn{2}{|c|}{ vZ Law } & \multicolumn{2}{|c|}{ ELR Law } & \multicolumn{3}{|c|}{ Combined } \\
\hline & & & Mean $\pm \sigma$ & Median $\pm \sigma_{\mathrm{g}}$ & Mean $\pm \sigma$ & Median $\pm \sigma_{\mathrm{g}}$ & Mean $\pm \sigma$ & Median $\pm \sigma_{\mathrm{g}}$ & $\sqrt{n}$ \\
\hline Nucleus members & 4 & 7 & $415 \pm 5$ & $415 \pm 6$ & $399 \pm 43$ & $404 \pm 55$ & $407 \pm 34$ & $414 \pm 35$ & 17 \\
\hline All members & 7 & 13 & $451 \pm 86$ & $415 \pm 71$ & $451 \pm 98$ & $408 \pm 110$ & $454 \pm 95$ & $415 \pm 93$ & 35 \\
\hline All excluding Chow & 6 & 11 & $416 \pm 11$ & $415 \pm 13$ & $424 \pm 79$ & $404 \pm 88$ & $421 \pm 59$ & $414 \pm 56$ & 23 \\
\hline
\end{tabular}

Note. $n$ is the number of stars in each subset and also corresponds to the number of age estimates in the vZ and ELR subsets. $n^{*}$ is the number of age estimates in the combined subsets and corresponds to $2 n-1$.
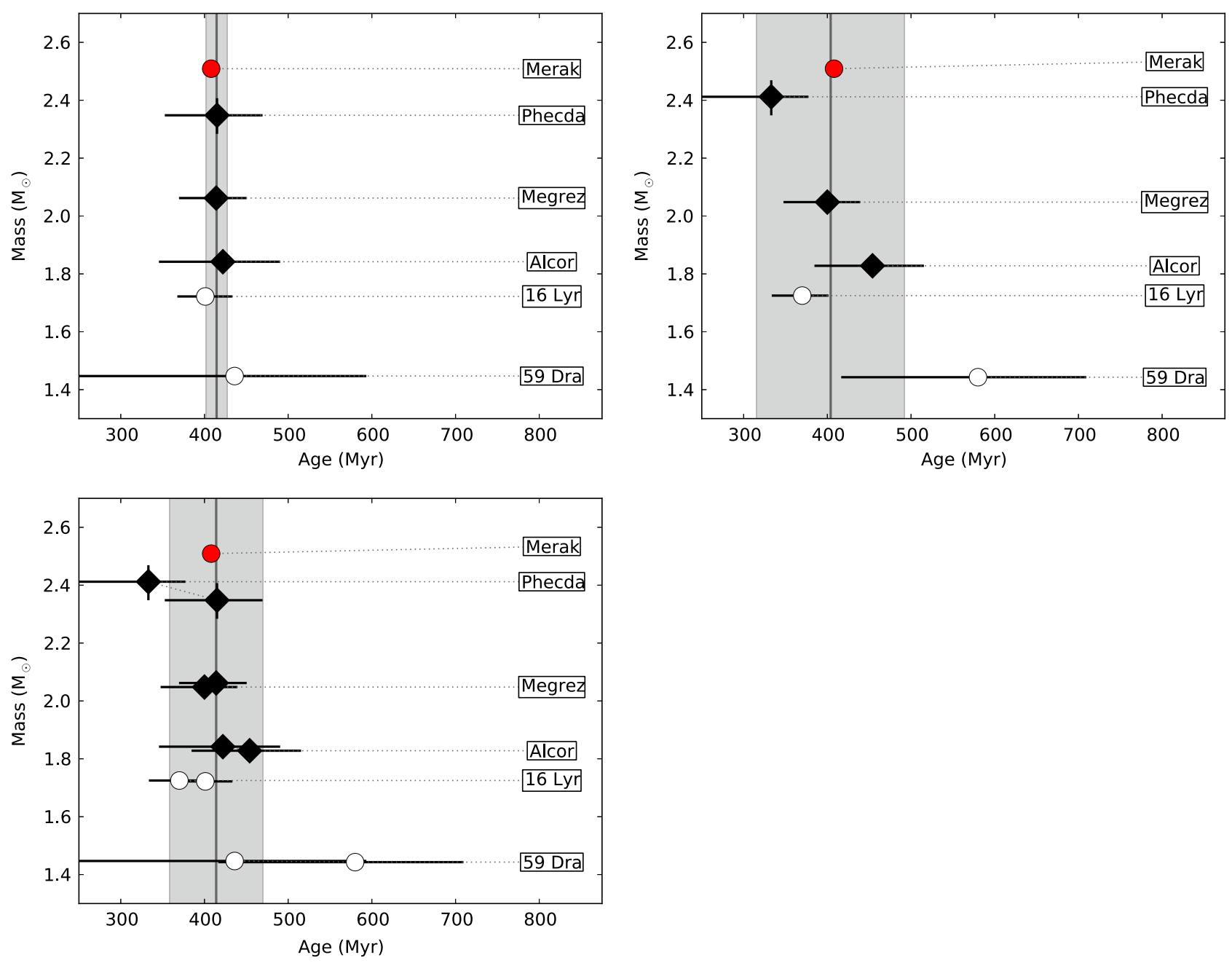

Figure 11. Same as Figure 10, but excluding Chow.

ages estimated using the vZ law than the ELR law. However, because this dispersion is of the same order of magnitude as the statistical uncertainties in the ages, we consider that this may be a statistical anomaly. The age estimated for Chow makes it older than the moving group as a whole and is thus excluded as a potential interloper in our final age estimate. Because neither gravity darkening law was favored, we combined the ages estimated with the vZ and ELR laws to determine the overall age of the moving group.

By determining the ages of these coeval stars, we validate this technique for use on individual field stars and determine a model uncertainty of approximately $10 \%$ for stars with masses ranging from $\sim 1.8$ to $2.5 M_{\odot}$. Using the independent technique described here, we find the age of the Ursa
Major moving group to be $414 \pm 23 \mathrm{Myr}$. This result is consistent with previous age estimates for the Ursa Major moving group.

The authors would like to thank John Monnier for his suggestions on how to construct the rapid rotator model and for providing test data, Michel Rieutord for his help in calculating $\beta$ for the ELR gravity darkening law, Doug Gies for his suggestions on how to handle model SEDs, and Brian Kloppenborg for his advice in making our model more computationally efficient. J.J. and R.W. acknowledge support from the NSF AAG grant number 1009643. T.S.B. acknowledged partial support from grants 12ADAP120172, 14XRP14_2-0147, and ADAP14-0245. 
Table 10

Adopted Photometry

\begin{tabular}{|c|c|c|c|c|c|c|c|}
\hline Bandpass & Merak & Phecda & Megrez & Alcor & Chow & $16 \mathrm{Lyr}$ & 59 Dra \\
\hline & HD 95418 & HD 103287 & HD 106591 & HD 116842 & HD 141003 & HD 177196 & HD 180777 \\
\hline \multicolumn{8}{|c|}{ Adopted Optical Photometry } \\
\hline \multicolumn{8}{|c|}{ Mermilliod 2006} \\
\hline Johnson $U$ & $2.349 \pm 0.014$ & $2.451 \pm 0.010$ & $3.460 \pm 0.009$ & $4.260 \pm 0.008$ & $3.816 \pm 0.023$ & $5.281 \pm 0.018$ & $5.440 \pm 0.016$ \\
\hline Johnson $B$ & $2.346 \pm 0.011$ & $2.437 \pm 0.006$ & $3.389 \pm 0.009$ & $4.176 \pm 0.007$ & $3.731 \pm 0.013$ & $5.199 \pm 0.015$ & $5.442 \pm 0.016$ \\
\hline Johnson $V$ & $2.366 \pm 0.009$ & $2.437 \pm 0.005$ & $3.312 \pm 0.007$ & $4.009 \pm 0.006$ & $3.667 \pm 0.010$ & $5.013 \pm 0.014$ & $5.136 \pm 0.014$ \\
\hline \multicolumn{8}{|c|}{ Hauck \& Mermilliod 1997} \\
\hline Strömgren $u$ & $3.741 \pm 0.022$ & $3.860 \pm 0.016$ & $4.849 \pm 0.011$ & $5.620 \pm 0.015$ & $5.261 \pm 0.029$ & 6.619 & 6.699 \\
\hline Strömgren $v$ & $2.501 \pm 0.012$ & $2.587 \pm 0.011$ & $3.572 \pm 0.010$ & $4.400 \pm 0.012$ & $3.922 \pm 0.019$ & 5.412 & 5.696 \\
\hline Strömgren $b$ & $2.349 \pm 0.006$ & $2.426 \pm 0.010$ & $3.350 \pm 0.010$ & $4.110 \pm 0.011$ & $3.715 \pm 0.012$ & 5.106 & 5.324 \\
\hline Strömgren $y$ & $2.355 \pm 0.005$ & $2.420 \pm 0.010$ & $3.312 \pm 0.010$ & $4.014 \pm 0.011$ & $3.670 \pm 0.010$ & 5.000 & 5.120 \\
\hline \multicolumn{8}{|c|}{ Infrared Photometry from Literature } \\
\hline \multicolumn{8}{|c|}{ Cutri et al. 2003} \\
\hline 2MASS $J$ & $2.269 \pm 0.244$ & $2.381 \pm 0.290$ & $3.316 \pm 0.248$ & $3.291 \pm 0.226$ & $3.440 \pm 0.290$ & $4.776 \pm 0.282$ & $4.338 \pm 0.222$ \\
\hline 2MASS $H$ & $2.359 \pm 0.164$ & $2.487 \pm 0.174$ & $3.306 \pm 0.252$ & $3.295 \pm 0.228$ & $3.539 \pm 0.276$ & $4.578 \pm 0.036$ & $4.264 \pm 0.144$ \\
\hline 2MASS $K$ & $2.285 \pm 0.244$ & $2.429 \pm 0.288$ & $3.104 \pm 0.338$ & $3.145 \pm 0.244$ & $3.546 \pm 0.318$ & $4.505 \pm 0.016$ & $4.313 \pm 0.018$ \\
\hline \multicolumn{8}{|c|}{ Ducati 2002} \\
\hline Johnson $K$ & $\ldots$ & $2.33 \pm 0.02$ & $\ldots$ & $1.76 \pm 0.01$ & $3.42 \pm 0.01$ & $\ldots$ & $\ldots$ \\
\hline \multicolumn{8}{|c|}{ Morel \& Magnenat 1978} \\
\hline Johnson $J$ & 2.350 & 2.400 & 3.110 & $\ldots$ & $\ldots$ & $\ldots$ & $\ldots$ \\
\hline Johnson $K$ & 2.350 & 2.370 & 3.090 & $\ldots$ & $\ldots$ & $\ldots$ & $\ldots$ \\
\hline \multicolumn{8}{|c|}{ Kidger \& Martin-Luis 2003} \\
\hline Johnson $J$ & $\ldots$ & $\ldots$ & $\ldots$ & $3.674 \pm 0.004$ & $\ldots$ & $\ldots$ & $\ldots$ \\
\hline Johnson $H$ & $\ldots$ & $\ldots$ & $\ldots$ & $3.623 \pm 0.004$ & $\ldots$ & $\ldots$ & $\ldots$ \\
\hline Johnson $K$ & $\cdots$ & $\cdots$ & $\cdots$ & $3.631 \pm 0.004$ & $\ldots$ & $\ldots$ & $\cdots$ \\
\hline \multicolumn{8}{|c|}{ Neugebauer \& Leighton 1969} \\
\hline Johnson $K$ & $2.38 \pm 0.06$ & $2.34 \pm 0.10$ & $\cdots$ & $\ldots$ & $\ldots$ & $\ldots$ & $\ldots$ \\
\hline \multicolumn{8}{|c|}{ Aumann \& Probst 1991} \\
\hline Johnson $J$ & $\ldots$ & $\ldots$ & $3.13 \pm 0.02$ & $\ldots$ & $\ldots$ & $\ldots$ & $\ldots$ \\
\hline Johnson $H$ & $\ldots$ & $\ldots$ & $3.10 \pm 0.02$ & $\ldots$ & $\ldots$ & $\ldots$ & $\ldots$ \\
\hline Johnson $K$ & $\ldots$ & $\ldots$ & $3.10 \pm 0.02$ & $\ldots$ & $\ldots$ & $\ldots$ & $\ldots$ \\
\hline \multicolumn{8}{|c|}{ Selby et al. 1988} \\
\hline Johnson $J$ & $\ldots$ & $\ldots$ & $\ldots$ & $3.77 \pm 0.03$ & $3.52 \pm 0.03$ & $\ldots$ & $\ldots$ \\
\hline Johnson $K$ & $\ldots$ & $\ldots$ & $\ldots$ & $3.63 \pm 0.03$ & $3.43 \pm 0.03$ & $\ldots$ & $\ldots$ \\
\hline \multicolumn{8}{|c|}{ Adopted Infrared Photometry } \\
\hline Johnson $J$ & $2.35 \pm 0.05$ & $2.40 \pm 0.05$ & $3.13 \pm 0.02$ & $3.674 \pm 0.004$ & $3.52 \pm 0.03$ & $\ldots$ & $\ldots$ \\
\hline Johnson $H$ & $\ldots$ & $\ldots$ & $3.10 \pm 0.02$ & $3.623 \pm 0.004$ & $\ldots$ & $\ldots$ & $\ldots$ \\
\hline Johnson $K$ & $2.38 \pm 0.06$ & $2.33 \pm 0.02$ & $3.10 \pm 0.02$ & $3.631 \pm 0.004$ & $3.42 \pm 0.01$ & & \\
\hline 2MASS $H$ & $\ldots$ & $\ldots$ & $\ldots$ & $\ldots$ & $\ldots$ & $\ldots$ & $4.264 \pm 0.144$ \\
\hline 2MASS $K$ & $\ldots$ & $\ldots$ & $\ldots$ & $\ldots$ & $\ldots$ & $\ldots$ & $4.313 \pm 0.03$ \\
\hline
\end{tabular}

Note. The photometric errors listed here are those reported by the respective surveys. We adopt errors of 0.03 mag for all photometric points with reported errors $<0.03$ mag (see Section 4.1). 


\section{APPENDIX PHOTOMETRY}

The photometry used to construct PEDs are presented in Table 10. The optical photometry is taken from Mermilliod (2006, UBV) and Hauck \& Mermilliod (1997, uvby). Values in these compilations are adopted over others because of the large number of observations that they average to compute final values, and because all eight stars are included in these surveys. Hauck \& Mermilliod (1997) do not report errors for Strömgren $y$ band measurements of Phecda and Megrez. For these stars, an error of $0.01 \mathrm{mag}$ is assumed, which is consistent with the photometric uncertainties of stars of similar brightness in their survey. In addition, Hauck \& Mermilliod (1997) do not report any uncertainties in Strömgren photometry for $16 \mathrm{Lyr}$ or 59 Dra. Near-infrared $(J H K)$ photometry are assembled from various sources and is either already in the Johnson photometric system or converted to it. 2MASS JHK photometry are listed in Table 10, but are not adopted for many of the sample stars because they are saturated.

Merak (HD 95418) - $J$ - and $K$-band photometry is adopted from Morel \& Magnenat (1978), and errors of $0.05 \mathrm{mag}$ are assumed. No $H$-band photometry is available.

Phecda (HD 103287) - J-band photometry is adopted from Morel \& Magnenat (1978), and errors of $0.05 \mathrm{mag}$ are assumed. $K$-band photometry is adopted from Ducati (2002). No $H$-band photometry is available.

Megrez (HD 106591) - J-, $H$-, and $K$-band photometry is adopted from Aumann \& Probst (1991), and the adopted uncertainties are the average uncertainties of that survey.

Alcor (HD 116842)- $J_{-}, H_{-}$, and $\mathrm{K}$-band photometry is adopted from Kidger \& Martin-Luis (2003) after converting the $J H K$ measurements found there to the Johnson system with the method found in Alonso et al. (1994). The adopted uncertainties are the average uncertainties of that survey.

Chow (HD 141003) - J-band photometry adopted from Selby et al. (1988) after converting the $J_{n}$ measurement found there to the Johnson system with the method found there. The adopted uncertainty is the reported uncertainty in that conversion. $K$-band photometry is adopted from Ducati (2002). No $H$-band photometry is available.

16 Lyr (HD 177196) - While 2MASS $K$-band photometry is unsaturated for this star, it is not adopted because including it causes the model to find a best fit with a $\chi^{2}$ in the visibility approximately double what it is without the $K$-band value. No $J$ - or $H$-band photometry is available.

59 Dra (HD 177196)-2MASS $H$ - and $K$-band photometry is unsaturated for this star, so it is adopted. No $J$-band photometry is available.

\section{REFERENCES}

Abt, H. A. 2009, AJ, 138, 28

Allen, C. W. C. W., \& Cox, A. N. 2000, Allen's astrophysical quantities (4th ed.; New York: AIP Press: Springer) mt Stromlo copy (barcode +2436171) accompanied by: 1 CD-ROM

Alonso, A., Arribas, S., \& Martinez-Roger, C. 1994, A\&AS, 107, 365

Ammler-von Eiff, M., \& Guenther, E. W. 2009, A\&A, 508, 677

Asiain, R., Figueras, F., Torra, J., \& Chen, B. 1999, A\&A, 341, 427

Aufdenberg, J. P., Mérand, A., Coudé du Foresto, V., et al. 2006, ApJ, 645, 664 Aumann, H. H., \& Probst, R. G. 1991, ApJ, 368, 264

Baron, F., Monnier, J. D., \& Kloppenborg, B. 2010, Proc. SPIE, 7734, 77342 Beers, T. C., Flynn, K., \& Gebhardt, K. 1990, AJ, 100, 32

Boden, A. F. 2007, NewAR, 51, 617

Boyajian, T. S. 2009, PhD thesis, Georgia State Univ.

Boyajian, T. S., McAlister, H. A., van Belle, G., et al. 2012, ApJ, 746, 101
Brandt, T. D., \& Huang, C. X. 2015, arXiv:1501.04404

Castelli, F., \& Kurucz, R. L. 2004, arXiv:astro-ph/0405087

Che, X., Monnier, J. D., Zhao, M., et al. 2011, ApJ, 732, 68

Claret, A. 2000, A\&A, 359, 289

Cox, A. N. 2000, Allen's Astrophysical Quantities (New York: AIP Press: Springer)

Cutri, R. M., Skrutskie, M. F., van Dyk, S., et al. 2003, yCat, 2246, 0

David, T. J., \& Hillenbrand, L. A. 2015, ApJ, 804, 146

De Rosa, R. J., Patience, J., Wilson, P. A., et al. 2014, MNRAS, 437, 1216

Ducati, J. R. 2002, yCat, 2237, 0

Eggen, O. J. 1992, AJ, 104, 1493

Espinosa Lara, F., \& Rieutord, M. 2011, A\&A, 533, A43

Galland, F., Lagrange, A.-M., Udry, S., et al. 2006, A\&A, 452, 709

Georgy, C., Ekström, S., Granada, A., et al. 2013, A\&A, 553, A24

Giannuzzi, M. A. 1979, A\&A, 77, 214

Girardi, L., Bertelli, G., Bressan, A., et al. 2002, A\&A, 391, 195

Glebocki, R., \& Gnacinski, P. 2005, yCat, 3244, 0

Gray, R. O., Corbally, C. J., Garrison, R. F., McFadden, M. T., \& Robinson, P. E. 2003, AJ, 126, 2048

Hauck, B., \& Mermilliod, M. 1997, yCat, 2215, 0

Henry, G. W., Fekel, F. C., \& Henry, S. M. 2007, AJ, 133, 1421

Husser, T.-O., Wende-von Berg, S., Dreizler, S., et al. 2013, A\&A, 553, A6

Ireland, M. J., Mérand, A., ten Brummelaar, T. A., et al. 2008, Proc. SPIE, 7013,24

Kalas, P., Graham, J. R., Chiang, E., et al. 2008, Sci, 322, 1345

Kidger, M. R., \& Martin-Luis, F. 2003, AJ, 125, 3311

King, J. R., \& Schuler, S. C. 2005, PASP, 117, 911

King, J. R., Villarreal, A. R., Soderblom, D. R., Gulliver, A. F., \& Adelman, S. J. 2003, AJ, 125, 1980

Kippenhahn, R., Weigert, A., \& Weiss, A. 2012, Stellar Structure and Evolution (Berlin: Springer)

König, B., Fuhrmann, K., Neuhäuser, R., Charbonneau, D., \& Jayawardhana, R. 2002, A\&A, 394, L43

Lafrasse, S., Mella, G., Bonneau, D., et al. 2010, Proc. SPIE, 7734, 77344

Levato, H., \& Abt, H. A. 1978, PASP, 90, 429

Maeder, A., \& Meynet, G. 2010, NewAR, 54, 32

Mamajek, E. E., \& Hillenbrand, L. A. 2008, ApJ, 687, 1264

Mamajek, E. E., Kenworthy, M. A., Hinz, P. M., \& Meyer, M. R. 2010, AJ, 139, 919

Marois, C., Macintosh, B., Barman, T., et al. 2008, Sci, 322, 1348

Marois, C., Zuckerman, B., Konopacky, Q. M., Macintosh, B., \& Barman, T. 2010, Natur, 468, 1080

McAlister, H. A., ten Brummelaar, T. A., Gies, D. R., et al. 2005, ApJ, 628, 439

Mermilliod, J. C. 2006, yCat, 2168, 0

Meynet, G., \& Maeder, A. 2000, A\&A, 361, 101

Monnier, J. D., Che, X., Zhao, M., et al. 2012, ApJL, 761, L3

Monnier, J. D., Zhao, M., Pedretti, E., et al. 2007, Sci, 317, 342

Morel, M., \& Magnenat, P. 1978, A\&AS, 34, 477

Moya, A., Amado, P. J., Barrado, D., et al. 2010, MNRAS, 405, L81

Neugebauer, G., \& Leighton, R. B. 1969, NASASP (Washington, D.C.: NASA)

Paxton, B., Bildsten, L., Dotter, A., et al. 2011, ApJS, 192, 3

Paxton, B., Cantiello, M., Arras, P., et al. 2013, ApJS, 208, 4

Perryman, M. A. C., Lindegren, L., Kovalevsky, J., et al. 1997, A\&A, 323, 49

Pourbaix, D. 2000, A\&AS, 145, 215

Roberts, L. C., Jr. 2011, MNRAS, 413, 1200

Roddier, F. 1981, Progress in Optics, Vol. 19 (Amsterdam: North-Holland)

Royer, F., Gebran, M., Monier, R., et al. 2014, A\&A, 562, A84

Royer, F., Zorec, J., \& Gómez, A. E. 2007, A\&A, 463, 671

Selby, M. J., Hepburn, I., Blackwell, D. E., et al. 1988, A\&AS, 74, 127

Soderblom, D. R., Pilachowski, C. A., Fedele, S. B., \& Jones, B. F. 1993, AJ, 105, 2299

ten Brummelaar, T. A., McAlister, H. A., Ridgway, S. T., et al. 2005, ApJ, 628,453

Ten Brummelaar, T. A., Sturmann, J., Ridgway, S. T., et al. 2013, JAI, 2, 1340004 Tomkin, J., \& Popper, D. M. 1986, AJ, 91, 1428

Torres, G., Andersen, J., \& Giménez, A. 2010, A\&AR, 18, 67

van Belle, G. T. 2012, A\&AR, 20, 51

van Belle, G. T., \& van Belle, G. 2005, PASP, 117, 1263

von Hoerner, S. 1957, ZAp, 42, 273

van Leeuwen, F. 2007, A\&A, 474, 653

von Zeipel, H. 1924a, MNRAS, 84, 665

von Zeipel, H. 1924b, MNRAS, 84, 684

Wainer, H., \& Thissen, D. 1976, Psychometrika, 41, 9

Zimmerman, N., Oppenheimer, B. R., Hinkley, S., et al. 2010, ApJ, 709, 733

Zorec, J., \& Royer, F. 2012, A\&A, 537, A120 